\title{
A GARCH option pricing model with filtered historical simulation
}

\author{
Barone-Adesi, Giovanni ; Engle, Robert F ; Mancini, Loriano
}

\begin{abstract}
We propose a new method for pricing options based on GARCH models with filtered historical innovations. In an incomplete market framework, we allow for different distributions of historical and pricing return dynamics, which enhances the model's flexibility to fit market option prices. An extensive empirical analysis based on SP 500 index options shows that our model outperforms other competing GARCH pricing models and ad hoc Black-Scholes models. We show that the flexible change of measure, the asymmetric GARCH volatility, and the nonparametric innovation distribution induce the accurate pricing performance of our model. Using a nonparametric approach, we obtain decreasing state-price densities per unit probability as suggested by economic theory and corroborating our GARCH pricing model. Implied volatility smiles appear to be explained by asymmetric volatility and negative skewness of filtered historical innovations.
\end{abstract}

DOI: https://doi.org/10.1093/rfs/hhn031

Posted at the Zurich Open Repository and Archive, University of Zurich

ZORA URL: https://doi.org/10.5167/uzh-10865

Journal Article

Accepted Version

Originally published at:

Barone-Adesi, Giovanni; Engle, Robert F; Mancini, Loriano (2008). A GARCH option pricing model with filtered historical simulation. Review of Financial Studies, 21(3):1223-1258.

DOI: https://doi.org/10.1093/rfs/hhn031 


\title{
A GARCH Option Pricing Model with Filtered Historical Simulation*
}

\author{
Giovanni Barone-Adesi ${ }^{\mathrm{a}}$ \\ Robert F. Engle ${ }^{b}$ \\ Loriano Mancini ${ }^{\mathrm{c}}$
}

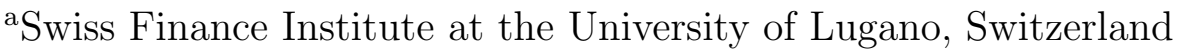

${ }^{\mathrm{b}}$ Dept. of Finance, Leonard Stern School of Business, New York University, NY

${ }^{\mathrm{c}}$ Swiss Banking Institute, University of Zurich, Switzerland

First draft: October 2004

This version: January 2008

${ }^{*}$ Correspondence Information: Giovanni Barone-Adesi, Swiss Finance Institute at the University of Lugano, Via Buffi 13, CH-6900 Lugano, Switzerland, E-mail address: baroneg@lu.unisi.ch. E-mail addresses for Robert Engle and Loriano Mancini: rengle@stern.nyu.edu, mancini@isb.uzh.ch. Financial support from the NCCR-FinRisk Swiss National Science Foundation (Barone-Adesi and Mancini) and the University Research Priority Program "Finance and Financial Markets" University of Zurich (Mancini) is gratefully acknowledged. For helpful comments we thank Yacine Aït-Sahalia (the editor), two anonymous referees, Fulvio Corsi, Robert Elliott, Jens Jackwerth and Claudia Ravanelli. 


\title{
A GARCH Option Pricing Model with Filtered Historical Simulation
}

\begin{abstract}
We propose a new method for pricing options based on GARCH models with filtered historical innovations. In an incomplete market framework, we allow for different distributions of historical and pricing return dynamics enhancing the model flexibility to fit market option prices. An extensive empirical analysis based on S\&P 500 index options shows that our model outperforms other competing GARCH pricing models and ad hoc Black-Scholes models. We show that the flexible change of measure, the asymmetric GARCH volatility and the nonparametric innovation distribution induce the accurate pricing performance of our model. Using a nonparametric approach, we obtain decreasing state price densities per unit probability as suggested by economic theory and corroborating our GARCH pricing model. Implied volatility smiles appear to be explained by asymmetric volatility and negative skewness of filtered historical innovations.
\end{abstract}

Keywords: Option pricing, GARCH model, state price density, Monte Carlo simulation.

JEL Classification: G13. 


\section{Introduction}

There is a general consensus that asset returns exhibit variances that change through time. GARCH models are a popular choice to model these changing variances - as is well documented in financial literature. However the success of GARCH in modeling historical return variances only partially extends to option pricing. Duan (1995) and Heston and Nandi (2000) among others assume normal return innovations and parametric risk premiums in order to derive GARCH-type pricing models. Their models consider historical and pricing (i.e. risk neutral) return dynamics in a unified framework. Unfortunately, they also imply that, up to the risk premium, conditional volatilities of historical and pricing distributions are governed by the same model parameters. Empirical studies, for instance by Chernov and Ghysels (2000) and Christoffersen and Jacobs (2004), show that this restriction leads to rather poor pricing and hedging performances. The reason is that changing volatility in real markets makes the perfect replication argument of Black and Scholes (1973) invalid. Markets are then incomplete in the sense that perfect replication of contingent claims using only the underlying asset and a riskless bond is impossible. Consequently, an investor would not necessarily price the option as if the distribution of its return had a different drift but unchanged volatility. Of course markets become complete if a sufficient (possibly infinite), number of contingent claims are available. In this case a well-defined pricing density exists. ${ }^{1}$

In the markets we consider, the volatility (and hence the distribution) of historical and pricing returns is different. This occurs because investors will set state prices to reflect their aggregate preferences. Our model differs from other models in financial literature because we rely on market incompleteness to allow for a pricing distribution different in shape from the historical distribution. It is possible then to calibrate the pricing process directly on option prices. Although this may appear to be a purely fitting exercise, involving no constraint beyond the absence of arbitrage,

\footnotetext{
${ }^{1}$ For instance Jarrow and Madan (1995) investigate the hedging of systematic jumps in asset returns when additional assets are introduced in the market.
} 
the stability of the pricing process over time and across maturities imposes substantial parameter restrictions. Furthermore, economic theory imposes further restrictions on investor preferences for aggregate wealth in different states, such as decreasing intertemporal marginal rate of substitutions. Pricing models are economically validated when these restrictions are satisfied.

This paper presents two main contributions: a new GARCH pricing model and the analysis of aggregate intertemporal marginal rate of substitutions. An in-depth empirical study underlies the previous contributions. Our GARCH pricing model relies on the Glosten, Jagannathan, and Runkle (1993) asymmetric volatility model driven by empirical GARCH innovations. The nonparametric distribution of innovations captures excess skewness, kurtosis and other nonstandard features of return data. We undertake an extensive empirical analysis using European options on the S\&P 500 Index from $1 / 2002$ to $12 / 2004$. We compare the pricing performances of our approach, the GARCH pricing models of Heston and Nandi (2000) and Christoffersen, Heston, and Jacobs (2006) and the benchmark model of Dumas, Fleming, and Whaley (1998). Interestingly, our GARCH pricing model outperforms all the other pricing methods in almost all model comparisons. We show that the flexible change of measure, the asymmetric GARCH volatility and the nonparametric innovation distribution induce the accurate pricing performance of our model. To economically validate our approach, we estimate the state price densities per unit probability (or aggregate intertemporal marginal rate of substitutions) for all the available maturities in our sample. Compared to previous studies, such as Jackwerth (2000) and Rosenberg and Engle (2002), we undertake a larger empirical analysis. More importantly, our estimates of the state price densities per unit probability tend to display the expected level and shape, as predicted by economic theory.

The financial literature on GARCH pricing models is rather extensive and we provide here only a partial overview. Heston and Nandi (2000) derive an almost closed form pricing formula assuming normal return innovations, a linear risk premium and the same GARCH parameters for historical and pricing asset returns. In our pricing model, we rely on Monte Carlo simulations and hence 
we can relax their assumptions. Duan (1996) calibrates a GARCH model to the FTSE 100 index options assuming Gaussian innovations and the locally risk neutral valuation relationship, i.e. same daily conditional variances under historical and pricing measures. Engle and Mustafa (1992) use a similar method calibrating a GARCH model to S\&P 500 index options to investigate the persistence of volatility shocks under historical and pricing distributions. Recent studies on GARCH pricing models include, for instance, Christoffersen, Heston, and Jacobs (2006) and Christoffersen, Jacobs, and Wang (2006), where option prices are computed using the technique in Heston and Nandi (2000), inverting moment generating functions.

The first section of this paper develops our theoretical framework, the second one presents our empirical findings on option pricing and state price density and the third one summarizes our conclusions.

\section{Theoretical framework}

\subsection{State price densities and derivative prices}

The relation between state price density (SPD), asset price dynamic and the representative agent's preferences is now well understood, but for completeness and to develop the notation, we provide a brief summary.

In a dynamic equilibrium model (such as Rubinstein (1976b) or Lucas (1978)), the price of any asset equals the expected present value of its future payoff, where the discounting is calculated using the riskless rate $r$ and the expectation is taken with respect to the probability density function (PDF) of the payoffs weighted by the marginal rate of substitution. This PDF is called the SPD or pricing density or risk neutral PDF (Cox and Ross (1976)) or equivalent martingale measure (Harrison and Kreps (1979)). Formally, the current price $\psi_{t}$ of an asset with single payoff $\psi_{T}$ at date $T=t+\tau$ is

$$
\psi_{t}=E_{\mathbb{Q}}\left[\psi_{T} e^{-r \tau} \mid \mathcal{F}_{t}\right]=e^{-r \tau} \int_{0}^{\infty} \psi_{T}\left(S_{T}\right) q_{t, T}\left(S_{T}\right) d S_{T}
$$


where $S_{T}$ is the state variable of the economy (e.g. aggregate consumption), $\mathcal{F}_{t}$ is the information available to the agent up to and including time $t, q_{t, T}$ is the SPD (the PDF under the risk neutral measure $\mathbb{Q}$ ) at time $t$ for payoffs liquidated at date $T$, and $E_{\mathbb{Q}}$ is the expectation under $\mathbb{Q}$. The security price $\psi_{t}$ can be equivalently represented as

$$
\psi_{t}=E_{\mathbb{P}}\left[\psi_{T} M_{t, T} \mid \mathcal{F}_{t}\right]=\int_{0}^{\infty} \psi_{T}\left(S_{T}\right) M_{t, T}\left(S_{T}\right) p_{t, T}\left(S_{T}\right) d S_{T}
$$

where $M_{t, T}$ is the SPD per unit probability ${ }^{2}$ and $p_{t, T}$ is the PDF under the historical or objective measure $\mathbb{P}$ at time $t$ for payoffs liquidated at date $T$. In a continuum of states, the SPD defines the Arrow-Debreu (Arrow (1964) and Debreu (1959)) security price. For each state of the economy, $s$, $q_{t, T}(s)$ is the forward price of an Arrow-Debreu security paying one dollar at time $T$ if the future state $S_{T}$ falls between $s$ and $s+d s$. The SPD per unit probability $M_{t, T}(s)$ is then the current market price of an Arrow-Debreu security per unit probability, with expected rate of return $1 / M_{t, T}(s)-1$ under the historical measure $\mathbb{P}$. Such expected rates of return depend on the current state of the economy summarized in the information set $\mathcal{F}_{t}$.

Equation (2) shows the high information content of the SPD per unit probability. This equation can be used to determine equilibrium asset prices given historical asset price dynamics and agent preferences, or to infer agent characteristics given the observed market asset prices. For instance, in a Lucas (1978) economy, the state variable $S_{T}$ is the aggregate consumption $C_{T}$ and $M_{t, T}=U^{\prime}\left(C_{T}\right) / U^{\prime}\left(C_{t}\right)$, that is the intertemporal marginal rate of substitution. Using an unconditional version of equation (2) and the aggregate consumption $C_{T}$ as state variable, Hansen and Singleton (1982) and Hansen and Singleton (1983) estimate risk aversion and time preference of the representative agent, hence identifying the SPD per unit probability. In general the SPD per unit

\footnotetext{
${ }^{2}$ The SPD per unit probability is also known as asset pricing kernel (Rosenberg and Engle (2002)) or stochastic discount factor; see Campbell, Lo, and MacKinlay (1997) and Cochrane (2001) for comprehensive surveys of its role in asset pricing, and Ross (1978), Harrison and Kreps (1979), Hansen and Richard (1987), Hansen and Jagannathan (1991) for related works.
} 
probability depends on all variables that affect marginal utility, such as past consumption or equity market returns; see for instance the discussion in Rosenberg and Engle (2002, Section 2.1) and references therein. Furthermore, due to the well-known measurement problems and the low temporal frequency of aggregate consumption data, ${ }^{3}$ several researchers have proposed alternative methods to estimate $M_{t, T}$ substituting consumption data with market data. For instance, Rosenberg and Engle (2002) project $M_{t, T}$ onto the payoffs of traded assets $\psi_{T}$, avoiding the issue of specifying the state variables in the SPD per unit probability. Aït-Sahalia and Lo (2000) and Jackwerth (2000) adopt similar approaches projecting $M_{t, T}$ onto equity return states using S\&P 500 index option prices. They assume that investors have a finite horizon and that the equity index level perfectly correlates with aggregate wealth. In this paper we also make the same assumptions. ${ }^{4}$ Our goal is to develop a pricing model for the derivative securities that takes into account the most important features of equity returns. The model will be evaluated on the basis of statistical measures (mispricing of existing securities) as well as economic measures (verifying whether SPD per unit probability satisfies economic criteria).

\subsection{Asset price dynamics}

In this section we develop the stochastic volatility model that captures the most important features of the equity return process. We use this model for pricing options.

\subsubsection{Historical return dynamics}

A substantial amount of empirical evidence suggest that equity return volatility is stochastic and mean reverting, return volatility responds asymmetrically to positive and negative returns, and return

\footnotetext{
${ }^{3}$ For discussions of these issues see for example Ferson and Harvey (1992), Wilcox (1992), and Slesnick (1998).

${ }^{4}$ See for example Rubinstein (1976a) and Brown and Gibbons (1985) for the conditions under which the SPD per unit probability with consumption growth rate as state variable is equivalent to a SPD per unit probability with equity index return as state variable.
} 
innovations are non-normal; see for instance Ghysels, Harvey, and Renault (1996). In a discrete time setting, the stochastic volatility is often modeled using extensions of the autoregressive conditional heteroscedasticity (ARCH) model proposed by Engle (1982). Comprehensive surveys of the ARCH and related models are Bollerslev, Chou, and Kroner (1992) and Bollerslev, Engle, and Nelson (1994). In a continuous time setting, stochastic volatility diffusion model are commonly used; surveys of this literature are for instance Ghysels, Harvey, and Renault (1996) and Shephard (1996).

To model the equity index return we use an asymmetric GARCH specification with an empirical innovation density. The GARCH model of Bollerslev (1986) accounts for stochastic, mean reverting volatility dynamics. The asymmetry term is based on Glosten, Jagannathan, and Runkle (1993) (GJR). The empirical innovation density captures potential non-normalities in the true innovation density and we refer to this approach as the filtering historical simulation (FHS) method. BaroneAdesi, Bourgoin, and Giannopoulos (1998) introduce the FHS method to compute portfolio risk measures and Engle and Gonzalez-Rivera (1991) investigate theoretical properties of the GARCH model with empirical innovations.

Under the historical measure $\mathbb{P}$ the asymmetric GJR GARCH model is

$$
\begin{aligned}
\log \left(S_{t} / S_{t-1}\right) & =\mu+\varepsilon_{t} \\
\sigma_{t}^{2} & =\omega+\beta \sigma_{t-1}^{2}+\alpha \varepsilon_{t-1}^{2}+\gamma I_{t-1} \varepsilon_{t-1}^{2},
\end{aligned}
$$

where $\varepsilon_{t}=\sigma_{t} z_{t}, z_{t} \sim f(0,1)$ and $I_{t-1}=1$, when $\varepsilon_{t-1}<0$ and $I_{t-1}=0$, otherwise. When $\gamma>0$ the model accounts for the leverage effect, ${ }^{5}$ i.e. bad news $\left(\varepsilon_{t-1}<0\right)$ raises the future volatility more than good news $\left(\varepsilon_{t-1} \geq 0\right)$ of the same absolute magnitude. The constant expected return $\mu$ of the log-return is not usually compatible with time varying state prices, but it is unlikely that

\footnotetext{
${ }^{5}$ The name leverage effect was introduced by Black (1976) who suggested that a large negative return increases the financial and operating leverage, and rises equity return volatility; see also Christie (1982). Campbell and Hentschel (1992) suggested an alternative explanation based on the market risk premium and volatility feedback effects; see also the more recent discussion by Bekaert and $\mathrm{Wu}(2000)$. We shall use the name leverage effect as it is commonly used by researchers when referring to the asymmetric reaction of volatility to positive and negative return innovations.
} 
it will strongly affect the estimation of state prices. Hence, equation (3) can be viewed as an approximation. The scaled return innovation $z_{t}$ is drawn from the empirical density function $f$ that is obtained by dividing each estimated return innovations $\hat{\varepsilon}_{t}$ by its estimated conditional volatility $\hat{\sigma}_{t}$. This set of estimated scaled innovations gives an empirical density function that incorporates excess skewness, kurtosis and other extreme return behaviors that are not captured in a normal density. We estimate the model parameters using the pseudo maximum likelihood (PML) approach (Bollerslev and Wooldridge (1992)) with the nominal (not necessarily true) assumption of normal innovations. This technique provides consistent parameter estimates even when the true innovation density $f$ is non-normal; see also Gourieroux, Monfort, and Trognon (1984).

The GARCH literature provides several specifications for the conditional volatility and we could adopt a different GARCH model in this study. We favor the asymmetric GJR GARCH model (3) mainly for two reasons: $(i)$ the flexibility in capturing the leverage effect and $(i i)$ the ability in fitting daily S\&P 500 index returns (used in our empirical application). Engle and Ng (1993) document that the GJR model provides an adequate modeling of the news impact curve, outperforming the EGARCH model of Nelson (1991). Rosenberg and Engle (2002) fit a number of GARCH models to daily S\&P 500 returns and they find that the GJR model (3) describes the data best. In Section 2 we undertake an extensive empirical analysis using several years of S\&P 500 returns and in some instances there might be other GARCH models which outperform the asymmetric GARCH model (3). For comparison purposes and to simplify implementation, we always maintain the GJR model in our study. If on specific occasions we use other GARCH models which fit the data better, we could obtain more accurate pricing results. Hence our findings can be interpreted in a conservative way. 


\subsubsection{Pricing return dynamics}

The asset return model (3) is specified under the historical measure $\mathbb{P}$ and cannot be used to price options directly. To achieve this goal, one possibility is to specify the SPD per unit probability (i.e. the change of measure from $\mathbb{P}$ to $\mathbb{Q}$ ) and to recover the pricing asset dynamics. ${ }^{6}$ This approach is particularly appealing because the GARCH parameter $\theta=\{\omega, \beta, \alpha, \gamma\}$ can be easily estimated using historical asset returns and then used for pricing purposes. Unfortunately, several studies (e.g. Chernov and Ghysels (2000), Christoffersen and Jacobs (2004) and our results in Table 9) show that this approach leads to a rather poor pricing performance and it is largely dominated by option pricing models calibrated only using option prices. ${ }^{7}$ This negative result is mainly due to the difficulties of specifying a correct SPD per unit probability, that in general has time varying level and shape; see for instance Rosenberg and Engle (2002).

We do not specify directly the change of measure from $\mathbb{P}$ to $\mathbb{Q}$, and we propose to approximate it by calibrating a new set of pricing GARCH parameter $\theta^{*}=\left\{\omega^{*}, \beta^{*}, \alpha^{*}, \gamma^{*}\right\}$ directly on market option prices. The parameter $\theta^{*}$ can possibly be different from the parameter $\theta$ under $\mathbb{P}$, and this difference characterizes the SPD per unit probability. We propose to calibrate the GJR pricing model using the FHS method and we call this approach FHS. ( $i$ ) On a given day $t$, the GARCH model (3) is estimated using $n$ historical log-returns of the underlying asset, $\left\{\log \left(S_{j} / S_{j-1}\right), j=1-n+t, 2-n+t, \ldots, t\right\}$. The empirical innovations $\left\{z_{j}=\varepsilon_{j} / \sigma_{j}\right\}$ are also estimated. (ii) Given the pricing GARCH parameters

\footnotetext{
${ }^{6}$ For instance Rubinstein (1976b) and Brennan (1979) originally used log-normal distributions and power utilities to characterize the SPD per unit probability, while Heston (1993b) and Stutzer (1996) combine log-exponential distributions with power utility and exponential utility for the same purpose.

${ }^{7}$ Although Chernov and Ghysels (2000) find that the pricing of long term options in the Heston (1993a) model could be improved using security and option contracts jointly and Christoffersen and Jacobs (2004) find that the outof-sample performances of certain GARCH models estimated on historical data are only slightly inferior to the ones of the same GARCH models calibrated only on option data.
} 
$\theta^{*}$, a return path from $t$ to $t+\tau$ is simulated using the GARCH pricing model for $i=t+1, \ldots, t+\tau$,

$$
\begin{aligned}
\log \left(S_{i} / S_{i-1}\right) & =\mu^{*}+\varepsilon_{i} \\
\sigma_{i}^{2} & =\omega^{*}+\beta^{*} \sigma_{i-1}^{2}+\alpha^{*} \varepsilon_{i-1}^{2}+\gamma^{*} I_{i-1} \varepsilon_{i-1}^{2},
\end{aligned}
$$

where the risk neutral drift $\mu^{*}$ ensures that the expected asset return equals the risk free rate, i.e. $E_{\mathbb{Q}}\left[S_{i} / S_{i-1} \mid \mathcal{F}_{i-1}\right]=e^{r}$. A return path is simulated by drawing an estimated past innovation, say, $z_{[1]}$, updating the conditional variance $\sigma_{t+1}^{2}$, drawing a second innovation $z_{[2]}$, updating the conditional variance $\sigma_{t+2}^{2}$, and so on up to $T=t+\tau$. The $\tau$ periods simulated return is $S_{T} / S_{t}=$ $\exp \left(\tau \mu^{*}+\sum_{i=1}^{\tau} \sigma_{t+i} z_{[i]}\right)$. (iii) The $\tau$ periods SPD is estimated by simulating several $\tau$ periods return paths, i.e. repeating the previous step several times. (iv) The price of a call option at time $t$ with strike $K$ and maturity $T$ is given by $e^{-r \tau} \sum_{l=1}^{L} \max \left(S_{T}^{(l)}-K, 0\right)$, where $S_{T}^{(l)}$ is the simulated asset price at time $T$ in the $l$-th sample path and $L$ is the total number of simulated sample paths (e.g. $L=20,000)$. Put prices are computed similarly. $(v)$ The pricing GARCH parameters $\theta^{*}$ are varied (which changes the sample paths) so as to best fit the cross section of option prices on date $t$, minimizing the mean square pricing error $\sum_{j=1}^{N_{t}} e\left(K_{j}, T_{j}\right)^{2}$, where $e\left(K_{j}, T_{j}\right)$ is the difference between the GARCH model price and the market price of the option $j$ with strike $K_{j}$ and maturity $T_{j} .{ }^{8} N_{t}$ is the number of options on a given day $t$. (vi) The calibration is achieved when, varying the pricing GARCH parameter $\theta^{*}$, the reduction in the mean square pricing error is negligible or below a given threshold.

To reduce the Monte Carlo variance, we use the empirical martingale simulation method proposed by Duan and Simonato (1998), where the simulated asset price paths are re-scaled to ensure that the risk neutral expectation of the underlying asset equals its forward price. To lighten the notation,

\footnotetext{
${ }^{8}$ To minimize the criterion function we use the Nelder-Mead simplex direct search method implemented in the Matlab function fminsearch, which does not require the computation of gradients. To ensure the convergence of the calibration algorithm, the FHS innovations used to simulate the GARCH sample paths are kept fix across all the iterations of the algorithm. Starting values for the pricing parameters $\theta^{*}$ are the GARCH parameters estimated under the historical measure $\mathbb{P}$ and obtained in the step $(i)$.
} 
$\sigma_{t}^{2}$ denotes the pricing as well as the historical volatility, but it will be clear from the context which volatility we are referring to. Similarly, $\varepsilon_{t}$ denotes pricing and historical raw innovation.

The distribution of the scaled innovation $z$ could also be changed to better approximate the change of measure from $\mathbb{P}$ to $\mathbb{Q}$. We retain the same historical distribution of the innovation process under the pricing measure $\mathbb{Q}$ because in our empirical applications the parameter change from $\theta$ to $\theta^{*}$ already provides a flexible change of measure to well fit market options prices. In Section 2.7 we investigate whether the induced SPD per unit probability is economically sustainable and satisfies usual economic restrictions on levels and shapes. In this case, the GARCH pricing model is validated economically.

If the return innovation $z$ was to be normal, the SPD per unit probability would be restricted as in Duan (1995, Lemma A.1) because both historical and pricing distributions would be normal and pricing asset returns could not follow a GARCH process with different parameters. ${ }^{9}$ Our pricing model does not rely on normal innovations, but on non-normal empirical innovations. To infer the pricing model, we do not transform the FHS innovation process $\left\{z_{t}\right\}$ and we exploit the flexibility of $\theta^{*}$ to compensate for that.

The previously mentioned calibration approach is usually undertaken in option pricing literature; see for instance Engle and Mustafa (1992), Duan (1996), Heston and Nandi (2000), and Christoffersen, Jacobs, and Wang (2006). ${ }^{10}$ Compared to previous studies, we use a different pricing model, we consider wider moneyness and/or maturity ranges of option prices and we undertake a more extensive calibration exercise, calibrating our GARCH pricing model and competing models each week from $1 / 2002$ to $12 / 2004$ (our sample data). Furthermore, we estimate the SPD per unit probability for all the available maturities in our database.

\footnotetext{
${ }^{9}$ We are grateful to the referee for pointing this out.

${ }^{10}$ See also Amin and Ng (1997) for related work on interest rate models.
} 


\section{Empirical analysis}

\section{$2.1 \quad$ Data}

To test our model we use European options on the S\&P 500 index (symbol: SPX). The market for these options is one of the most active index options market in the world. Expiration months are the three near-term months and three additional months from the March, June, September, December, quarterly cycle. Strike price intervals are 5 and 25 points. The options are European and have no wild card features. SPX options can be hedged using the active market on the S\&P 500 futures. Consequently, these options have been the focus of many empirical investigations, including AïtSahalia and Lo (1998), Chernov and Ghysels (2000), Heston and Nandi (2000), and Carr, Geman, Madan, and Yor (2003).

We consider closing prices of the out-of-the-money (OTM) put and call SPX options for each Wednesday $^{11}$ from January 2, 2002 to December 29, 2004. It is known that OTM options are more actively traded than in-the-money options and using only OTM options avoids potential issues associated with liquidity problems. ${ }^{12}$ Option data and all the other necessary data are downloaded from OptionMetrics. The average of bid and ask prices are taken as option prices, while options with time to maturity less than 10 days or more than 360 days, implied volatility larger than $70 \%$, or prices less than $\$ 0.05$ are discarded, which yields a sample of 29,211 observations. Put and call options are equally represented in the sample, that is $50.7 \%$ and $49.3 \%$, respectively.

Using the term structure of zero-coupon default-free interest rates, the riskless interest rate for each given maturity $\tau$ is obtained by linearly interpolating the two interest rates whose maturities

\footnotetext{
${ }^{11}$ In our sample all but two days are Wednesdays. In those cases we take the subsequent trading day, but for simplicity we refer to all days as Wednesdays.

${ }^{12}$ Daily volumes of out-of-the-money put options are usually several times as large as volumes of in-the-money puts. This phenomenon started after the October 1987 crash and reflects the strong demand by portfolio managers for protective puts, inducing implied volatility smiles.
} 
straddle $\tau$. This procedure is repeated for each contract and each day in our sample.

We divide the option data into several categories according to either time to maturity or moneyness, $m$, defined as the ratio of the strike price over the asset price, $K / S$. A put option is said to be deep out-of-the-money if its moneyness $m<0.85$, or out-of-the-money if $0.85 \leq m<1$. A call option is said to be out-of-the-money if $1 \leq m<1.15$; and deep out-of-the-money if $m \geq 1.15$. An option contract can be classified by the time to maturity, as short maturity ( $<60$ days); medium maturity (60-160 days); and long maturity ( $>160$ days).

Table 1 describes the 29,211 option prices, the implied volatilities, and the bid-ask spreads in our database. The average put (call) prices range from $\$ 0.77$ (\$0.34) for short maturity, deep OTM options to $\$ 38.80$ (\$34.82) for long maturity, OTM options. OTM put and call options account for $27 \%$ and $25 \%$, respectively, of the total sample. Short and long maturity options account for $33 \%$ and $36 \%$, respectively, of the total sample. The table also shows the volatility smile and the corresponding term structure. For each given set of maturities the smile across moneyness is evident. When the time to maturity increases the smile tends to become flatter and the bid-ask spreads tend to narrow. The number of options on each Wednesday is on average 186.1, with a standard deviation of 22.3, a minimum of 142 and a maximum of 237 option contracts.

During our sample period, the $\mathrm{S} \& \mathrm{P} 500$ index ranges from a minimum of $\$ 776.8$ to a maximum of $\$ 1,213.5$ with an average level of $\$ 1,029.5$. The average daily log-return is quite close to zero $\left(6.6 \times 10^{-5}\right)$, the standard deviation is $22.98 \%$ on an annual base, ${ }^{13}$ and skewness and kurtosis are 0.25 and 4.98 respectively.

\footnotetext{
${ }^{13}$ The standard deviation of the S\&P 500 log-returns is approximately in line with the GARCH unconditional volatility estimates reported in Tables 2 and 3.
} 


\subsection{Benchmark model and alternative GARCH pricing models}

In the empirical analysis we compare our GARCH pricing model to three competing approaches: the benchmark ad hoc Black-Scholes model proposed by Dumas, Fleming, and Whaley (1998) (BS, in brief), the GARCH model with Gaussian innovations of Heston and Nandi (2000) (HN, in brief), and the GARCH model with Inverse Gaussian innovations of Christoffersen, Heston, and Jacobs (2006) (IG, in brief).

The benchmark ad hoc Black-Scholes model is estimated as follows. Implied volatilities of the cross section of the SPX options are smoothed across strikes and time to maturities by fitting the following function

$$
\sigma^{\mathrm{bs}}=a_{0}+a_{1} K+a_{2} K^{2}+a_{3} \tau+a_{4} \tau^{2}+a_{5} K \tau
$$

where $\sigma^{\text {bs }}$ is the Black-Scholes implied volatility for an option with strike $K$ and time to maturity $\tau$. Option prices are then obtained by plugging in the Black-Scholes formula the fitted implied volatilities. Equation (5) is estimated using ordinary least squares (OLS). Although theoretically inconsistent, ad hoc Black-Scholes methods are routinely used in the option pricing industry and they represent a more challenging benchmark than the simple Black-Scholes model, because they allow for different implied volatilities to price different options. Indeed, Dumas, Fleming, and Whaley (1998) show that this approach outperforms the deterministic volatility function option valuation model introduced by Derman and Kani (1994), Dupire (1994), and Rubinstein (1994). ${ }^{14}$

Heston and Nandi (2000) apply the inversion of the characteristic function technique introduced by Heston (1993a) to derive an almost closed form expression for European option prices under GARCH models with Gaussian innovations. To capture the well documented negative asymmetry in GARCH innovations, Christoffersen, Heston, and Jacobs (2006) extend the previous technique to GARCH models with possibly negative skewed inverse Gaussian innovations. Here we recall

\footnotetext{
${ }^{14}$ See for instance Buraschi and Jackwerth (2001) for a discussion on this point.
} 
the pricing formulae and the specification of the two GARCH models. We refer the reader to the corresponding papers for an in-depth discussion of the models.

In the HN model, the asset return dynamic under the risk neutral measure $\mathbb{Q}$ is

$$
\begin{aligned}
\log \left(S_{t} / S_{t-1}\right) & =r-\sigma_{t}^{2} / 2+\sigma_{t} z_{t} \\
\sigma_{t}^{2} & =\omega_{\mathrm{hn}}^{*}+\beta_{\mathrm{hn}}^{*} \sigma_{t-1}^{2}+\alpha_{\mathrm{hn}}^{*}\left(z_{t-1}-\gamma_{\mathrm{hn}}^{*} \sigma_{t-1}\right)^{2}
\end{aligned}
$$

where $z_{t}$ is a standard Gaussian innovation, and in the IG model

$$
\begin{aligned}
\log \left(S_{t} / S_{t-1}\right) & =r+\nu \sigma_{t}^{2}+\eta^{*} y_{t} \\
\sigma_{t}^{2} & =w^{*}+b^{*} \sigma_{t-1}^{2}+c^{*} y_{t-1}+a^{*} \sigma_{t-1}^{4} / y_{t-1},
\end{aligned}
$$

where $y_{t}$ follows an inverse Gaussian distribution with parameter $\delta_{t}=\sigma_{t}^{2} / \eta^{* 2}$. In both models, at time $t$ the call option $C_{t}$ with strike price $K$ and time to maturity $\tau$ is worth

$$
C_{t}=e^{-r \tau} \zeta^{*}(1)\left(\frac{1}{2}+\frac{1}{\pi} \int_{0}^{\infty} \Re\left[\frac{K^{-i \phi} \zeta^{*}(i \phi+1)}{i \phi \zeta^{*}(1)}\right] d \phi\right)-e^{-r \tau} K\left(\frac{1}{2}+\frac{1}{\pi} \int_{0}^{\infty} \Re\left[\frac{K^{-i \phi} \zeta^{*}(i \phi)}{i \phi}\right] d \phi\right),
$$

where $\Re[\cdot]$ denotes the real part of a complex number, $\zeta^{*}(\phi)$ is the conditional moment generating function at time $t$ of the $\log$-price $X_{T}=\log \left(S_{T}\right)$,

$$
\zeta^{*}(\phi)=E_{\mathbb{Q}}\left[e^{\phi X_{T}} \mid \mathcal{F}_{t}\right]=S_{t}^{\phi} e^{A_{t}+B_{t} \sigma_{t+1}^{2}}
$$

The coefficients $A_{t}$ 's and $B_{t}$ 's are computed backward starting from the terminal condition $A_{T}=$ $B_{T}=0$, and using the following recursive equations in the HN model

$$
\begin{aligned}
& A_{t}=A_{t+1}+\phi r+B_{t+1} \omega_{\mathrm{hn}}^{*}-\frac{1}{2} \log \left(1-2 \alpha_{\mathrm{hn}}^{*} B_{t+1}\right) \\
& B_{t}=\phi\left(\gamma_{\mathrm{hn}}^{*}-\frac{1}{2}\right)-\frac{\gamma^{*} \mathrm{hn}}{2}+\beta_{\mathrm{hn}}^{*} B_{t+1}+\frac{1 / 2\left(\phi-\gamma_{\mathrm{hn}}^{*}\right)^{2}}{1-2 \alpha_{\mathrm{hn}}^{*} B_{t+1}}
\end{aligned}
$$

and in the IG model

$$
\begin{aligned}
& A_{t}=A_{t+1}+\phi r+B_{t+1} w^{*}-\frac{1}{2} \log \left(1-2 a^{*} \eta^{* 4} B_{t+1}\right) \\
& B_{t}=B_{t+1} b^{*}+\phi \nu^{*}+\eta^{*-2}-\eta^{*-2} \sqrt{\left(1-2 a^{*} \eta^{* 4} B_{t+1}\right)\left(1-2 c^{*} B_{t+1}-2 \eta^{*} \phi\right)} .
\end{aligned}
$$


The motivation for choosing the BS, HN and IG models for comparison is as follows. The three models allow to ascertain whether introducing changing volatility (not allowed in BS) or nonnormality (not allowed in BS and HN) leads to better pricing using our FHS approach. The comparison between IG and FHS models concerns the relative advantage of modeling non-normality of innovations parametrically or in a nonparametric way, respectively.

\subsection{Calibration of the pricing models}

All the pricing models (FHS, HN, IG and BS) are calibrated on the cross section of out-of-themoney SPX option prices. This procedure is repeated each Wednesday from 1/2002 to $12 / 2004$, thus obtaining 157 calibrations for each model.

To calibrate our FHS model, the GARCH model (3) is estimated each Wednesday using the PML approach and $n=3,500$ historical log-returns, i.e. a sample size of approximately fourteen years. Unreported means and standard deviations of FHS innovations are remarkably close to zero and one, respectively, meaning that GJR GARCH models are correctly identified. FHS innovations are highly non-normal and descriptive statistics are available from the authors upon request. As an example, Figure 1 shows the S\&P 500 log-returns, the estimated GARCH volatility $\left\{\sigma_{t}\right\}$ and the scaled innovations $\left\{z_{t}\right\}$ up to a randomly chosen date, July 9, 2003. A sufficiently long sample size ensures that the innovation distribution is adequately estimated. As a robustness check, we repeated all the estimations and calibration exercises using a sample size $n=2,500$; this point is discussed in Section 2.5. The GARCH pricing parameter $\theta^{*}=\left\{\omega^{*}, \beta^{*}, \alpha^{*}, \gamma^{*}\right\}$ in model (4) is calibrated using the FHS method described in Section 1.2.2. The current conditional volatility $\sigma_{t+1}$ is readily obtained using historical GARCH estimates and asset returns. This is an important advantage of the GARCH pricing models over continuous time stochastic volatility models where the current instantaneous volatility is not observable and is usually calibrated on option prices increasing the computational burden; see for instance Bakshi, Cao, and Chen (1997). The SPD is estimated by simulating $L$ 
$=20,000$ return paths. ${ }^{15}$ The sample paths are simulated in parallel running only one for-loop, ${ }^{16}$ on a standard Pentium IV processor with 1GB RAM. Hence our Monte Carlo approach could be potentially inaccurate - as any other simulation method - but the computation time is roughly the same as for the competing GARCH pricing models, where one for-loop is needed to compute the coefficients $A_{t}$ 's and $B_{t}$ 's. ${ }^{17}$ Whether Monte Carlo option prices are sufficiently accurate for pricing purposes will be reflected in the empirical pricing performance of our approach. However, for almost all the deepest out-of-the-money options, at least 100 simulated paths end "in-the-money". As an example, Figure 2 shows the calibration of the GJR model with FHS method on the cross-section of out-of-the-money SPX options on July 9, 2003. Given the wide range of moneyness and maturities considered, the calibration is quite satisfactory. An extensive empirical analysis is undertaken in the next sections.

When computing option prices using the HN and IG models, the integrals in equation (6) are computed numerically via discretization. We take as integration domain the interval $(0,100)$ and we evaluate the integrand function on 5,000 equally spaced mid-points. Then, the integrals are computed by averaging the function values over such an interval. ${ }^{18}$ As the moment generating function $\zeta^{*}$ does not depend on the strike $K$, for each given maturity $\tau$, the coefficients $A_{t}$ 's and $B_{t}$ 's need to be computed only once. Hence, this numerical procedure allows to evaluate a whole cross section of option prices in a few seconds, largely reducing the calibration time. As in Christoffersen, Heston,

\footnotetext{
${ }^{15}$ For a few selected days we repeated the calibration exercises using $L=10,000$ sample paths and we obtained very similar pricing results. As the computation burden was still manageable we used $L=20,000$ sample paths in our empirical study.

${ }^{16}$ The for-loop is the programming language statement which allows code to be repeatedly executed.

${ }^{17}$ If the number of simulated sample paths $L$ has to be increased and the computer memory is not enough then the Monte Carlo approach is substantially slower than the competing GARCH approaches.

${ }^{18}$ The choice of the integration domain and the subinterval length are selected to match option prices on a few selected days computed using the Romberg's numerical integration method over the interval $\left(10^{-6}, 200\right)$ with tolerance $10^{-4}$. Some differences between the two approaches are observed for only a few deepest out-of-the-money option prices.
} 
and Jacobs (2006), we set the riskless rate $r=0.05 / 365$ in the GARCH pricing formula. At each step of the calibration procedure, the conditional variances $\sigma^{2}$ is initialized at the unconditional variance level and then updated using the corresponding pricing GARCH dynamic. The iterations are started 250 trading days before the first option date to allow for the models to find the right conditional variances.

Christoffersen, Heston, and Jacobs (2006) show that the $\nu^{*}$ parameter in the IG model is not a free parameter but it is constrained to ensure that the underlying asset earns the risk free rate under the risk neutral measure $\mathbb{Q}$. This observation explains why $\nu^{*}$ does not appear in Table 3 which shows the calibrated risk neutral parameters.

Finally, when implementing all the pricing formulae, the dividends paid by the stocks in the S\&P 500 index have to be taken into account. Dividends are treated in different ways in option pricing literature; see for instance Aït-Sahalia and Lo (1998) and Heston and Nandi (2000) for two alternative procedures. We use the dividend yields downloaded from OptionMetrics to compute an ex-dividend spot index level.

\subsection{In-sample model comparison}

Table 3 shows summary statistics for the pricing GARCH parameters calibrated each Wednesday from $1 / 2002$ to $12 / 2004$. It is known that option prices are more sensitive for example to $\gamma^{*}$, $\gamma_{\mathrm{hn}}^{*}$, $c^{*}, \eta^{*}$ than $\omega^{*}, \omega_{\mathrm{hn}}^{*}, w^{*}$. Indeed, the first set of parameters turn out to be more stable than the second one, confirming the finding for instance in Heston and Nandi (2000). As expected, all the unconditional volatilities and the corresponding persistency measures are more stable than single parameters. For each Wednesday, we also calibrate the ad hoc Black-Scholes model (5) and Table 4 shows summary statistics for the parameter estimates $a_{i}, i=0, \ldots, 5$. The low standard deviations of the parameter estimates confirm that the implied volatility smile is a persistent phenomenon in SPX option market. 
Following Dumas, Fleming, and Whaley (1998) and Heston and Nandi (2000), in order to assess the quality of the pricing models, we report several measurements of fit. The dollar root mean square error (RMSE) is the square root of the averaged squared deviations between the model prices and the market prices; the mean absolute error (MAE) is the average of the absolute valuation error when the model price is outside the bid-ask spread; the mean outside error (MOE) is the average of the valuation error when the model price is outside the bid-ask spread; the minimum and maximum pricing errors; the percentage of positive pricing errors; the average pricing error as a percentage of the bid-ask spread.

For all pricing models, Table 5 shows the in-sample pricing errors summarized by the previous measurements of fit. Table 6 disaggregates the in-sample pricing errors by moneyness/maturity categories; see also the left graphs in Figure 3 for the in-sample absolute mispricing. The overall conclusion is that in terms of all measurements of fit our GJR model with FHS innovations outperforms all the other pricing models. For example, aggregate RMSE of IG and HN models are $46 \%$ and $37 \%$ larger than the RMSE of our FHS model. Occasionally, the GJR is somewhat outperformed by other models (for e.g. in terms of RMSE by the HN model during 2003 in Table 5, or by the IG model for deep out-of-the-money put options in Table 6), but its good pricing performance is remarkably stable. The percentage of positive pricing errors in Table 5 for the HN and the IG models seems to be too low, but no systematic bias emerges for the MOE's in Table 6 and hence this finding is due to slight underestimations of option prices. Finally, all the GARCH pricing models largely outperform the benchmark ad hoc Black-Scholes model, which has difficulties in fitting the whole cross section of option prices. As a further comparison, we also consider CGMY models proposed by Carr, Geman, Madan, and Yor (2003), where the underlying asset follows a mean corrected, time-changed exponential Lévy processes. Despite their models having more parameters (for e.g. CGMYSA model has nine parameters), our FHS model compares favorably. To save space the corresponding results are omitted but are available from the authors upon request. 


\subsection{Out-of-sample model comparison}

Out-of-sample forecasting of option prices is an interesting challenge for any pricing method. It tests not only the goodness-of-fit of the pricing formulae, but also whether the pricing methods overfit the option prices in the in-sample period. For each Wednesday in our sample, in-sample model estimates are used to price SPX options one week later (hence out-of-sample) using asset prices, time to maturities and interest rates relevant on the next Wednesday. ${ }^{19}$

Table 7 shows the out-of-sample pricing errors summarized by the different measurements of fit and Table 8 disaggregates the out-of-sample pricing errors by moneyness/maturity categories; see also the right graphs in Figure 3 for the out-of-sample absolute mispricing. Interestingly, the outof-sample results largely confirm the previous in-sample ones and overall our GJR model with FHS innovations outperforms all the competing pricing models. Hence our model is flexible enough to achieve a good pricing performance, capturing the pricing mechanisms, without overfitting the data.

In all the previous in- and out-of-sample pricing exercises the GJR GARCH is estimated and calibrated using $n=3,500$ past $\mathrm{S} \& \mathrm{P} 500$ log-returns. As a robustness check, we repeat all the previous analyses using $n=2,500$ log-returns. We obtain very similar results in terms of parameter estimation, calibration and pricing performance. These additional findings confirm the robustness of the reported results. The corresponding tables are omitted but are available from the authors.

\subsection{Analysis of GJR GARCH pricing model with FHS method}

\subsubsection{News impact curves}

It might be surprising that the GJR GARCH with FHS innovations outperforms the other GARCH pricing models, sometimes even by a large extent. After all, also the HN and IG models can account for the same stylized facts about the asset returns and volatility dynamics. To shed light on the

\footnotetext{
${ }^{19}$ The one week ahead forecast horizon is also adopted by Dumas, Fleming, and Whaley (1998) and Heston and Nandi (2000), among others, and for comparative purposes we adopt it here as well.
} 
differences among the GARCH models, we compute the news impact curve (Engle and Ng (1993)) implied by the different models. The news impact curve (NIC) measures the impact on the conditional variance $\sigma^{2}$ of a shock in the scaled innovation $z$, when the volatility is at its long run level. Figure 4 shows the NIC for the FHS, HN and IG models using the pricing GARCH parameters in Table 3. The graphs have to be interpreted carefully because the NIC is estimated using the average pricing parameter values and to simplify the interpretation, the vertical axis shows the conditional volatility rather than the conditional variance. However, the findings are remarkably stable across the three years and show that different GARCH volatilities react to innovation shocks differently. Overall, negative shocks $z$ tend to raise volatilities more than positive shocks of equal magnitude (with some exceptions for the IG model), documenting leverage effects. This effect is much stronger in the FHS than HN model. Gaussian innovations in the HN model are very rarely below, say, -3 , while in the GJR model with FHS, innovations are often below this threshold; see for instance Figure 1. The IG model can potentially react very strongly to negative shocks because the inverse Gaussian innovation, $y_{t}$, enters as $1 / y_{t}$ in the GARCH dynamics. Hence NIC tends to explode when $y_{t}$ goes to zero, although the probability of this event depends on the degrees of freedom parameter $\delta_{t}=\sigma_{t}^{2} / \eta^{* 2}$.

\subsubsection{Comparison to alternative GJR GARCH models}

To further understand the pricing performance of our FHS model, we compare it to the following two GARCH models: the GJR model fitted to historical returns and driven by FHS innovations (HIST, in brief), and the GJR model calibrated to the cross section of SPX options and driven by Gaussian innovations (GAUSS, in brief). The calibration procedure of the GAUSS model is the same as the FHS model but the FHS innovations are replaced by randomly drawn Gaussian innovations. ${ }^{20}$ As in

\footnotetext{
${ }^{20}$ To reduce the variance of the Monte Carlo estimates we use the method of antithetic variates; see, for instance, Boyle, Broadie, and Glassermann (1997). This method is not used in our FHS approach so as to preserve the negative asymmetry of the historical GARCH innovations.
} 
previous analysis, GARCH models are re-estimated and re-calibrated each Wednesday from 1/2002 to 12/2004; see Table 2 for GARCH parameters of HIST and GAUSS models. Table 9 shows in- and out-of-sample aggregated pricing results; to simplify comparisons, pricing results of HN and FHS models are reported in this table as well. Comparing FHS and HIST models (which rely on the same FHS innovation distribution but different asset return dynamics) shows that allowing for different historical and pricing return distributions induces a major improvement in the pricing performance, such as a reduction of more than $70 \%$ in terms of RMSE. Comparing FHS and GAUSS models (which rely on the same GARCH dynamic but different innovation distributions) shows that using nonparametric instead of Gaussian innovations induces an overall improvement in pricing options, but by a lesser extent. Finally, comparing GAUSS and HN models (which rely on different GARCH dynamics but the same Gaussian innovation distribution) shows that the GJR GARCH dynamic allows for a better fitting of option prices than the asymmetric GARCH dynamic in Heston and Nandi (2000). Pricing errors disaggregated across years and moneyness/maturity categories confirm the results in Table 9 and are omitted, but are available from the authors upon request.

Overall, allowing for different volatilities (and hence different distributions) under historical and pricing measures induces a major improvement in the pricing performance. This finding is in line with recent studies on variance swap contracts, which document higher risk neutral volatilities than historical ones, inducing negative volatility premia; see for instance Carr and Wu (2007) and Egloff, Leippold, and $\mathrm{Wu}$ (2007). Capturing this phenomenon seems to be crucial in order to achieve accurate pricing performances.

\subsubsection{Applications of GJR GARCH pricing model with FHS method}

The proposed GARCH pricing model has been used to price European calls and puts but the model has other potential applications as well. As the calibration procedure relies on Monte Carlo simulation, transition densities of the stock price are readily available. Hence other European and 
path-dependent claims can be priced and hedged using the simulated trajectories of the stock price. In particular, a sensitivity analysis (e.g. computing delta and gamma) can be easily obtained following the direct approach in Duan (1995) or the regression methodology approach in Longstaff and Schwartz (2001). Recently, Barone-Adesi and Elliott (2007) proposed a model-free approach to compute delta hedge ratios. Comparing option price forecasts based on their model-free approach and on our GARCH model allows to evaluate the deterioration of delta hedging due to volatility shocks.

Our GARCH pricing model provides an arbitrage free procedure to optimally interpolate strike prices and time to maturities, as required in several applications. ${ }^{21}$ In particular, the grid of time to maturities is rather sparse and standard interpolation methods are likely not to work in that case.

Our FHS model also provides risk neutral dynamic and forecast of the pricing volatility term structure. Such forecasts are not available in other models, such as ad hoc Black-Scholes methods, but play a key role in pricing and hedging volatility-dependent claims. To gauge the accuracy of the GARCH forecasts based on the FHS method, for each Wednesday $t$ from 1/2002 to 12/2004, Figure 8 shows the VIX, a 30-day volatility index constructed by the Chicago Board of Options Exchange, and the conditional forecast of the risk neutral integrated volatility,

$$
100 \times \sqrt{\frac{365}{30} \sum_{i=1}^{30} E_{\mathbb{Q}}\left[\sigma_{t+i}^{2} \mid \mathcal{F}_{t}\right]},
$$

given by our GARCH pricing model. In most occasions the VIX and GARCH forecasts are very close, the correlation is 0.96 , the average difference (GARCH forecast minus VIX) is $0.1 \%$, and the standard deviation of such differences is $1.9 \%$. An in-depth analysis of GARCH volatility forecasts is beyond the scope of this paper but these findings suggest that our GARCH pricing model can provide sensible predictions of the risk neutral volatility term structure.

\footnotetext{
${ }^{21}$ Interpolation methods are used for instance to synthesize variance swap rates from European calls and puts; see Carr and $\mathrm{Wu}(2007)$.
} 


\subsection{State Price Density per unit probability}

In this section we estimate SPD per unit probability, $M_{t, t+\tau}$, using our FHS model (Section 1.2) and the GAUSS model (Section 2.6.2), and we study their economic implications. Estimates of $M_{t, t+\tau}$ are based on conditional historical and pricing densities, $p_{t, t+\tau}$ and $q_{t, t+\tau}$. In our FHS model, the historical density $p_{t, t+\tau}$ is obtained by simulating from $t$ to $t+\tau$ the GJR model fitted to historical log-returns up to time $t$ and driven by FHS innovations. ${ }^{22}$ The historical density depends on the expected return, which is notoriously difficult to estimate. Indeed, Merton (1980) argues that positive risk premia should be explicitly modeled and, as in Jackwerth (2000), we set the risk premium at $8 \%$ per year. The pricing density $q_{t, t+\tau}$ is obtained by simulating the GJR model calibrated on the crosssection of SPX options observed on date $t$ and driven by the same FHS innovations. Then, $M_{t, t+\tau}$ is given by the discounted ratio of the pricing over the historical densities, $M_{t, t+\tau}=e^{-r \tau} q_{t, t+\tau} / p_{t, t+\tau}$. This procedure gives semiparametric estimates of $M_{t, t+\tau}$, because the densities $p_{t, t+\tau}$ and $q_{t, t+\tau}$ are based on parametric GARCH models, but no a priori functional form is imposed on $M_{t, t+\tau}$. In the GAUSS model, the estimation procedure of $M_{t, t+\tau}$ is the same as for the FHS model but the FHS innovations are replaced by Gaussian innovations.

We estimate SPD per unit probability for each Wednesday $t$ and each time to maturity $\tau$ in our sample from 1/2002 to $12 / 2004$, using both GJR GARCH models with FHS and Gaussian innovations. We obtain 862 density estimates of $M_{t, t+\tau}$ for each model. As an example, Figure 5 shows historical and pricing densities on July 9, 2003, using our FHS approach and SPD per unit probability for different times to maturities estimated using GJR models with the FHS and Gaussian innovations; see also Table 10 for the numerical values of $M_{t, t+\tau}$. In agreement with economic theory, all state price densities per unit probability are fairly monotonically decreasing in $S_{T}$, with some exceptions for the longest maturity and the lowest states. Interestingly, SPD per unit probabilities

\footnotetext{
${ }^{22}$ All density functions are estimated using the Matlab function ksdensity with Gaussian kernels and optimal default bandwidths for estimating Gaussian densities.
} 
based on FHS innovations are lower than those based on Gaussian innovations; this point is discussed below. To visually represent all SPD per unit probabilities, we follow a similar approach as in Jackwerth (2000). For each Wednesday $t$ and maturity $\tau, M_{t, t+\tau}$ 's are first estimated on a grid of 100 points of the gross return $\left\{S_{t+\tau} / S_{t}\right\}$, and then averaged across each year and each short, medium and long maturity categories. The averaged SPD per unit probability, and \pm 0.5 times the empirical standard deviations, are plotted in Figure 6. As predicted by economic theory and in contrast to previous studies such as Jackwerth (2000) and Rosenberg and Engle (2002), our estimates of SPD per unit probability are monotonically decreasing in the S\&P 500 state variable. ${ }^{23}$ Moreover, estimates of $M_{t, t+\tau}$ based on Gaussian innovations are clearly higher than those based on FHS innovations for low states, but very close for medium and high states of the S\&P 500. These differences are economically significant. High values of $M_{t, t+\tau}$ on the left imply that simple Arrow-Debreu state contingent claims are "overpriced" and have very negative expected rates of return. As an example, Table 11 shows average SPD per unit probabilities and implied expected returns of Arrow-Debreu securities for long maturities (> 160 days) in 2002 as defined in our sample (Section 2.1). ArrowDebreu securities for low states of S\&P 500 can be viewed as "insurance" contracts, providing positive payoff when the economy is in a bad state. Hence, not surprisingly, investors are prepared to suffer expected losses when entering these contracts. However, such expected losses might appear "unreasonably" high when SPD per unit probabilities are estimated using Gaussian innovations, such as $1 / 4.20-1=-76.2 \%$, for moneyness of 0.40 . In contrast, SPD per unit probability estimated using our FHS method implies an expected rate of return of $1 / 1.50-1=-33.3 \%$ for the same contingent claim, which is certainly a more "reasonable" return. As a consequence, $M_{t, t+\tau}$ 's based on Gaussian innovations imply that out-of-the-money puts appear to be "overpriced", while using FHS innovations they are not. Negative skewness of FHS innovations and asymmetric GARCH

\footnotetext{
${ }^{23}$ In this paper we use a different methodology, sample period and moneyness/maturity ranges for the SPX options than previously mentioned studies. These differences can explain the different findings.
} 
volatilities can account for excess out-of-the-money put prices and provide an adequate pricing of the downside market risk.

To assess the time stability of SPD per unit probabilities, Figure 7 shows estimates of $M_{t, t+\tau}$ on four consecutive Wednesdays in July 2002, July 2003 and July 2004, and for time to maturity $\tau$ closest to 100 days. Similar qualitative findings are observed for other periods and time to maturities. SPD per unit probability under both FHS and Gaussian innovations tends to change slowly through time, although our FHS method provides more stable estimates. ${ }^{24}$ This finding is consistent with aggregate risk aversion (absolute slope of SPD per unit probability) changing slowly through time at a given wealth level. For example, Rosenberg and Engle (2002) document that empirical risk aversions change with the business cycle in a counter-cyclical way, increasing (decreasing) during contractions (expansions) of the economy.

Comparing Tables 2 and 3 shows that the asymmetry parameter $\gamma^{*}$ tends to be larger when the GJR model is calibrated using Gaussian innovation rather than FHS innovations. Hence, despite the stochastic volatility, Gaussian innovations cannot account for market expectations on frequencies of large negative returns and to fit out-of-the-money put prices, a large asymmetry parameter $\gamma^{*}$ is needed. Using FHS innovations, the GJR model can more easily price out-of-the-money puts giving SPD per unit probability more in line with economic reasonings.

Several other studies provide estimates of SPD per unit probability. Perhaps the most closely related study is provided by Rosenberg and Engle (2002). The main similarity is that in both cases conditional SPD per unit probability is investigated, reflecting current market conditions such as high or low volatility. The main difference is that Rosenberg and Engle (2002) consider a flexible but parametric functional form for $M_{t, t+\tau}$, while here we provide nonparametric estimates of the ratio $q_{t, t+\tau} / p_{t, t+\tau}$. Detlefsen, Härdle, and Moro (2007) estimate conditional SPD per unit probability

\footnotetext{
${ }^{24}$ The rather volatile estimates of SPD per unit probability for lowest moneyness correspond to low pricing errors in the calibration procedure and hence they have a limited impact on calibrated models.
} 
nonparametrically using DAX options data, but relying on the Heston (1993a) volatility model. Aït-Sahalia and Lo (2000) and Jackwerth (2000) provide nonparametric estimates of SPD per unit probability (or related quantities such as risk aversions), but they exploit the time continuity of $M_{t, t+\tau}$ to average state prices and probabilities across time. Hence, their results are best interpreted as estimates of the average SPD per unit probability over the sample period, instead of conditional estimates; for a recent extension see Constantinides, Jackwerth, and Perrakis (2007). Compared to all the previous studies, we provide estimates of SPD per unit probability for many more time horizons $\tau$ and much larger ranges of $S_{t+\tau} / S_{t}$.

\section{Conclusions}

We propose a new method for pricing options based on an asymmetric GARCH model with filtered historical innovations. In an incomplete market framework, we allow for historical and pricing distributions to have different shapes, enhancing the model flexibility to fit market option prices. An extensive empirical analysis based on S\&P 500 index options shows that our model outperforms other competing GARCH pricing models and ad hoc Black-Scholes methods. We show that the flexible change of measure, the asymmetric GARCH volatility dynamic and the nonparametric innovation distribution induce the accurate pricing performance of our model. In contrast to previous studies, using our GARCH model and a nonparametric approach we obtain decreasing state price densities per unit probability as suggested by economic theory therefore validating our GARCH pricing model. Furthermore, implied volatility smiles appear to be explained by asymmetric GARCH volatility and negative skewness of the filtered historical innovations, with no need for "unreasonably" high state prices for out-of-the-money puts. Our discrete-time model does not impose restrictions similar to Girsanov's theorem on the change of measure. The only requirement is the non-negativity of state prices, necessary for no arbitrage and ensured by our Monte Carlo method. Such a weak require- 
ment allows for more flexible option pricing than enabled by diffusion models. Further refinements of pricing and stability issues are left to future research. 


\section{References}

Aït-Sahalia, Y., and A. W. Lo, 1998, "Nonparametric estimation of state-price densities implicit in financial assets prices," Journal of Finance, 53, 499-548.

— , 2000, "Nonparametric risk management and implied risk aversion," Journal of Econometrics, $94,9-51$.

Amin, K. I., and V. K. Ng, 1997, "Inferring future volatility from the information in implied volatility in Eurodollar options: A new approach," Review of Financial Studies, 10, 333-367.

Arrow, K., 1964, "The role of securities in the optimal allocation of risk bearing," Review of Economic Studies, 31, 91-96.

Bakshi, G., C. Cao, and Z. Chen, 1997, "Empirical performance of alternative option pricing models," Journal of Finance, 52, 2003-2049.

Barone-Adesi, G., F. Bourgoin, and K. Giannopoulos, 1998, "Don't look back," Risk, 11, 100-103.

Barone-Adesi, G., and R. J. Elliott, 2007, "Cutting the hedge," Computational Economics, 29, 151158.

Bekaert, G., and G. Wu, 2000, "Asymmetric volatility and risk in equity markets," Review of Financial Studies, 13, 1-42.

Black, F., 1976, "Studies of stock market volatility changes," in Proceedings of the 1976 Meetings of the American Statistical Association, Business and Economic Statistic Section, pp. 177-181.

Black, F., and M. Scholes, 1973, "The valuation of options and corporate liabilities," Journal of Political Economy, 81, 637-654.

Bollerslev, T., 1986, "Generalized autoregressive conditional heteroskedasticity," Journal of Econometrics, 31, 307-327. 
Bollerslev, T., R. Y. Chou, and K. F. Kroner, 1992, "ARCH modeling in finance - a review of the theory and empirical evidence," Journal of Econometrics, 52, 5-59.

Bollerslev, T., R. F. Engle, and D. B. Nelson, 1994, "ARCH models," in Handbook of Econometrics, ed. by R. F. Engle, and D. L. McFadden. North-Holland, Amsterdam, pp. 2959-3038.

Bollerslev, T., and J. M. Wooldridge, 1992, "Quasi-maximum likelihood estimation and inference in dynamic models with time varying covariances," Econometric Reviews, 11, 143-172.

Boyle, P., M. Broadie, and P. Glassermann, 1997, "Monte Carlo methods for security pricing," Journal of Economic Dynamics and Control, 21, 1267-1321.

Brennan, M. J., 1979, "The pricing of contingent claims in discrete time models," Journal of Finance, $34,53-68$.

Brown, D. P., and M. R. Gibbons, 1985, "A simple econometric approach for utility-based asset pricing models," Journal of Finance, 40, 359-381.

Buraschi, A., and J. C. Jackwerth, 2001, "The price of a smile: Hedging and spanning in option markets," Review of Financial Studies, 14, 495-527.

Campbell, J. Y., and L. Hentschel, 1992, "No news is good news: An asymmetric model of changing volatility in stock returns," Journal of Financial Economics, 31, 281-318.

Campbell, J. Y., A. W. Lo, and C. MacKinlay, 1997, The econometrics of financial markets. Princeton University Press, Princeton, NY.

Carr, P., H. Geman, D. B. Madan, and M. Yor, 2003, "Stochastic volatility for Lévy processes," Mathematical Finance, 13, 345-382.

Carr, P., and L. Wu, 2007, "Variance risk premia," Review of Financial Studies, forthcoming. 
Chernov, M., and E. Ghysels, 2000, "A study towards a unified approach to the joint estimation of objective and risk neutral measures for the purpose of options valuation," Journal of Financial Economics, 56, 407-458.

Christie, A., 1982, "The stochastic behavior of common stock variances: Value, leverage and interest rate effects," Journal of Financial Economics, 10, 407-432.

Christoffersen, P., S. Heston, and K. Jacobs, 2006, "Option valuation with conditional skewness," Journal of Econometrics, 131, 253-284.

Christoffersen, P., and K. Jacobs, 2004, "Which GARCH model for option valuation?," Management Science, 50, 1204-1221.

Christoffersen, P., K. Jacobs, and Y. Wang, 2006, "Option valuation with long-run and short-run volatility components," working paper, McGill University, Canada.

Cochrane, J. H., 2001, Asset pricing. Princeton University Press, Princeton.

Constantinides, G. M., J. C. Jackwerth, and S. Perrakis, 2007, "Mispricing of S\&P 500 index options," Review of Financial Studies, forthcoming.

Cox, J. C., and S. A. Ross, 1976, "The valuation of options for alternative stochastic processes," Journal of Financial Economics, 3, 145-166.

Debreu, G., 1959, Theory of value. Wiley, New York.

Derman, E., and I. Kani, 1994, "Riding on the smile," Risk, 7, 32-39.

Detlefsen, K., W. K. Härdle, and R. A. Moro, 2007, "Empirical pricing kernels and investor preferences," working paper, Humboldt University of Berlin.

Duan, J.-C., 1995, "The GARCH Option Pricing Model," Mathematical Finance, 5, 13-32. 
— 1996, "Cracking the Smile," Risk, 9, 55-59.

Duan, J.-C., and J.-G. Simonato, 1998, "Empirical martingale simulation for asset prices," Management Science, 44, 1218-1233.

Dumas, B., J. Fleming, and R. E. Whaley, 1998, "Implied volatility functions: Empirical tests," Journal of Finance, 53, 2059-2106.

Dupire, B., 1994, "Pricing with a smile," Risk, 7, 18-20.

Egloff, D., M. Leippold, and L. Wu, 2007, "Variance risk dynamics, variance risk premia, and optimal variance swap investments," working paper, Baruch College.

Engle, R. F., 1982, "Autoregressive conditional heteroscedasticity with estimates of the variance of United Kingdom inflation," Econometrica, 50, 987-1007.

Engle, R. F., and G. Gonzalez-Rivera, 1991, "Semiparametric ARCH models," Journal of Business and Economic Statistics, 9, 345-359.

Engle, R. F., and C. Mustafa, 1992, "Implied ARCH Models from Options Prices," Journal of Econometrics, 52, 289-311.

Engle, R. F., and V. K. Ng, 1993, "Measuring and testing the impact of news on volatility," Journal of Finance, 48, 1749-1778.

Ferson, W. E., and C. R. Harvey, 1992, "Seasonality and consumption-based asset pricing," Journal of Finance, 47, 511-552.

Ghysels, E., A. C. Harvey, and E. Renault, 1996, "Stochastic volatility," in Handbook of Statistics, ed. by G. S. Maddala, and C. R. Rao. North-Holland, Amsterdam, pp. 119-191.

Glosten, L. R., R. Jagannathan, and D. E. Runkle, 1993, "On the relation between the expected value and the volatility of the nominal excess return on stocks," Journal of Finance, 48, 1779-1801. 
Gourieroux, C., A. Monfort, and A. Trognon, 1984, "Pseudo maximum likelihood methods: Theory," Econometrica, 52, 681-700.

Hansen, L. P., and R. Jagannathan, 1991, "Implications of security market data for models of dynamic economies," Journal of Political Economy, 99, 225-262.

Hansen, L. P., and S. Richard, 1987, "The role of conditioning information in deducing testable restrictions implied by asset pricing models," Econometrica, 55, 587-613.

Hansen, L. P., and K. J. Singleton, 1982, "Generalized instrumental variables estimation of nonlinear rational expectations models," Econometrica, 50, 1269-1286.

_ 1983, "Stochastic consumption, risk aversion, and the temporal behavior of asset returns," Journal of Political Economy, 91, 249-265.

Harrison, M., and D. Kreps, 1979, "Martingales and arbitrage in multiperiod securities markets," Journal of Economic Theory, 20, 381-408.

Heston, S., 1993a, "A closed-form solution for options with stochastic volatility, with applications to bond and currency options," Review of Financial Studies, 6, 327-343.

, 1993b, "Invisible parameters in option prices," Journal of Finance, 48, 933-947.

Heston, S., and S. Nandi, 2000, "A closed-form GARCH option valuation model," Review of Financial Studies, 13, 585-625.

Jackwerth, J. C., 2000, "Recovering risk aversion from option prices and realized returns," Review of Financial Studies, 13, 433-451.

Jarrow, R., and D. B. Madan, 1995, "Option pricing using the term structure of interest rates to hedge systematic discontinuities in asset returns," Mathematical Finance, 5, 311-336. 
Longstaff, F., and E. S. Schwartz, 2001, "Valuing American options by simulations: A simple leastsquares approach," Review of Financial Studies, 14, 113-147.

Lucas, R., 1978, "Asset prices in an exchange economy," Econometrica, 46, 1429-1446.

Merton, R. C., 1980, "On estimating the expected return on the market: An exploratory investigation," Journal of Financial Economics, 8, 323-361.

Nelson, D. B., 1991, "Conditional heteroskedasticity in asset returns: A new approach," Econometrica, 59, 347-370.

Rosenberg, J. V., and R. F. Engle, 2002, "Empirical pricing kernels," Journal of Financial Economics, $64,341-372$.

Ross, S. A., 1978, "A simple approach to the valuation of risky streams," Journal of Business, 51, 453-475.

Rubinstein, M., 1976a, "The strong case for the generalized logarithmic utility model as the premier model of financial markets," Journal of Finance, 31, 551-571.

_ 1976b, "The valuation of uncertain income streams and the pricing of options," Bell Journal of Economics, 7, 407-425.

— , 1994, "Implied binomial trees," Journal of Finance, 49, 771-818.

Shephard, N., 1996, "Statistical aspects of ARCH and stochastic volatility," in Time Series Models in Econometrics, Finance, and other Fields, ed. by D. R. Cox, O. E. Barndorff-Nielsen, and D. V. Hinkley. Chapman \& Hall, London, pp. 1-67.

Slesnick, D. T., 1998, "Are our data relevant to the theory? The case of aggregate consumption," Journal of the American Statistical Association, 16, 52-61. 
Stutzer, M., 1996, "A simple nonparametric approach to derivative security valuation," Journal of Finance, 51, 1633-1652.

Wilcox, D., 1992, "The construction of U.S. consumption data: Some facts and their implications for empirical work," American Economic Review, 82, 922-941. 


\begin{tabular}{|c|c|c|c|c|c|c|c|}
\hline \multirow{3}{*}{$\begin{array}{c}\text { Moneyness } \\
K / S\end{array}$} & & \multicolumn{6}{|c|}{ Maturity } \\
\hline & & \multicolumn{2}{|c|}{ Less than 60} & \multicolumn{2}{|c|}{60 to 160} & \multicolumn{2}{|c|}{ More than 160} \\
\hline & & Mean & Std. & Mean & Std. & Mean & Std. \\
\hline \multirow{4}{*}{$<0.85$} & Put price $\$$ & 0.77 & 1.20 & 2.54 & 3.20 & 8.69 & 7.63 \\
\hline & $\sigma^{\mathrm{bs}} \%$ & 38.97 & 9.59 & 33.36 & 7.75 & 28.37 & 5.21 \\
\hline & Bid-Ask\% & 0.99 & 0.69 & 0.69 & 0.68 & 0.27 & 0.36 \\
\hline & Observations & 1,798 & & 2,357 & & 2,845 & \\
\hline \multirow[t]{4}{*}{$0.85-1.00$} & Put price $\$$ & 8.43 & 7.60 & 19.12 & 11.63 & 38.80 & 15.67 \\
\hline & $\sigma^{\mathrm{bs}} \%$ & 22.38 & 7.05 & 21.88 & 5.35 & 21.28 & 3.91 \\
\hline & Bid-Ask\% & 0.19 & 0.21 & 0.10 & 0.05 & 0.06 & 0.03 \\
\hline & Observations & 3,356 & & 2,136 & & 2,314 & \\
\hline \multirow[t]{4}{*}{$1.00-1.15$} & Call price $\$$ & 7.45 & 7.81 & 15.79 & 12.60 & 34.82 & 18.19 \\
\hline & $\sigma^{\mathrm{bs}} \%$ & 17.55 & 5.80 & 17.31 & 5.02 & 17.61 & 4.00 \\
\hline & Bid-Ask\% & 0.34 & 0.46 & 0.17 & 0.23 & 0.07 & 0.04 \\
\hline & Observations & 2,955 & & 2,128 & & 2,247 & \\
\hline \multirow[t]{4}{*}{$>1.15$} & Call price $\$$ & 0.34 & 0.43 & 0.85 & 1.61 & 3.96 & 5.60 \\
\hline & $\sigma^{\mathrm{bs}} \%$ & 34.87 & 12.35 & 24.34 & 8.27 & 18.87 & 4.48 \\
\hline & Bid-Ask\% & 1.81 & 0.47 & 1.49 & 0.71 & 0.83 & 0.78 \\
\hline & Observations & 1,633 & & 2,288 & & 3,154 & \\
\hline
\end{tabular}

Table 1: Mean, standard deviation (Std.) and number of observations for each moneyness/maturity category of out-of-the-money SPX options observed on Wednesdays from January 2, 2002 to December 29, 2004, after applying the filtering criteria described in the text. $\sigma^{\text {bs }}$ is the Black-Scholes implied volatility. Bid-Ask\% is $100 \times($ ask price bid price)/market price, where the market price is the average of the bid and ask prices. Moneyness is the strike price divided by the spot asset price, $K / S$. Maturity is measured in calendar days. 


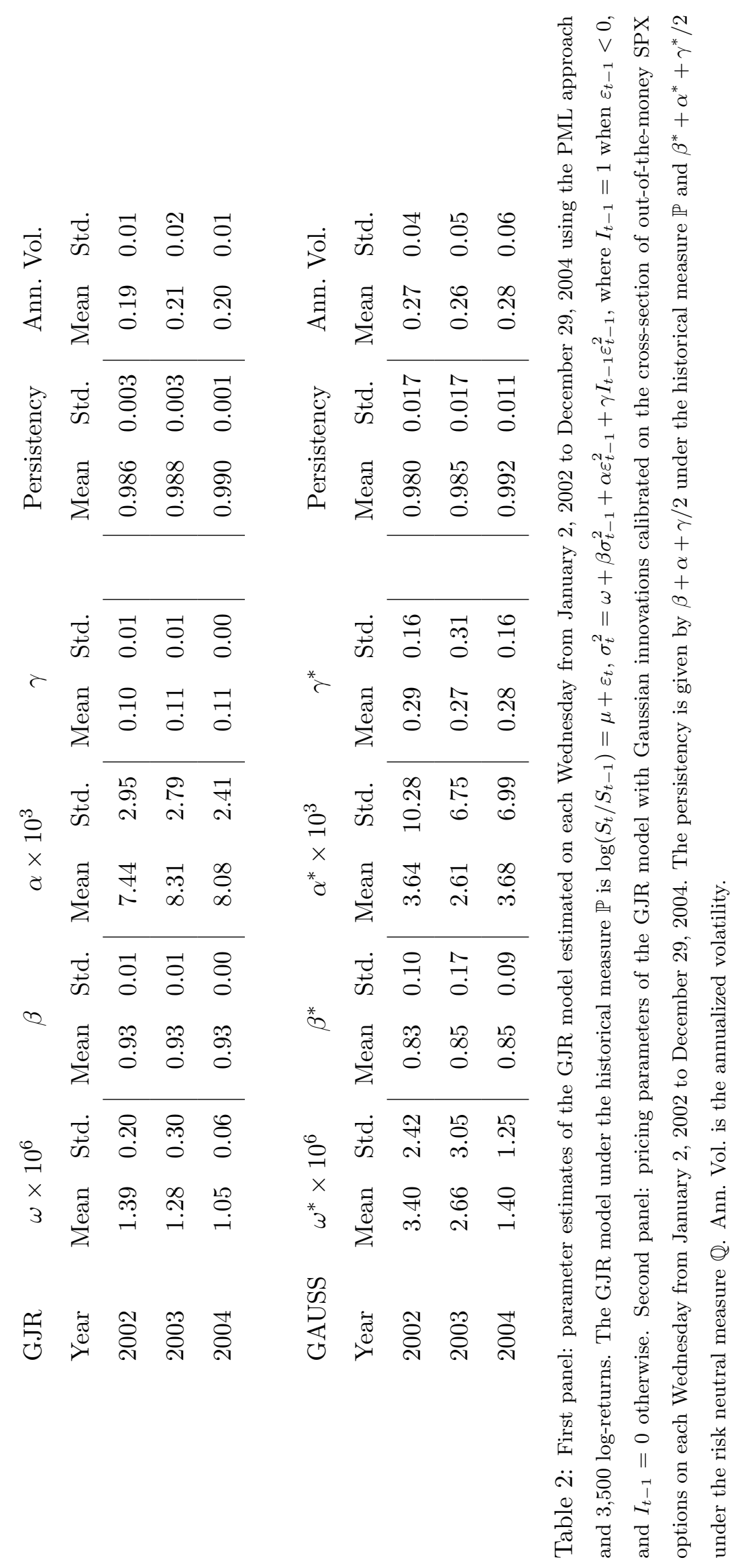




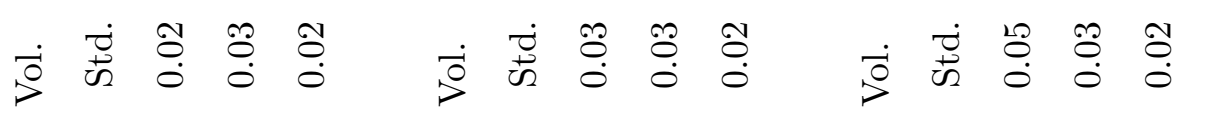

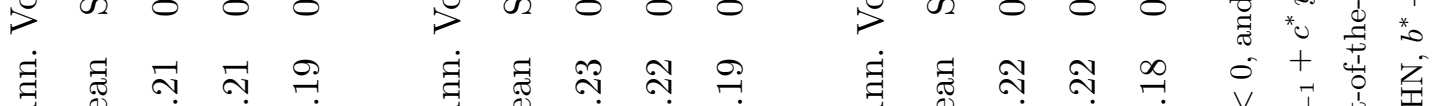

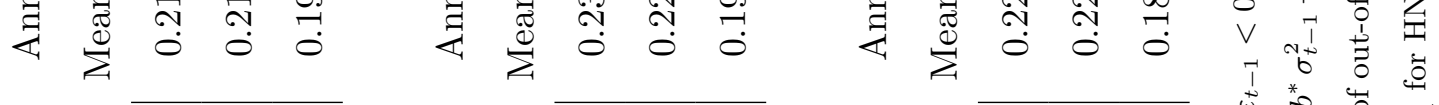

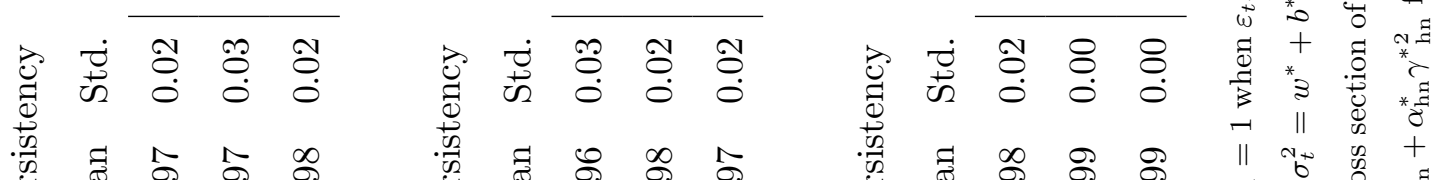

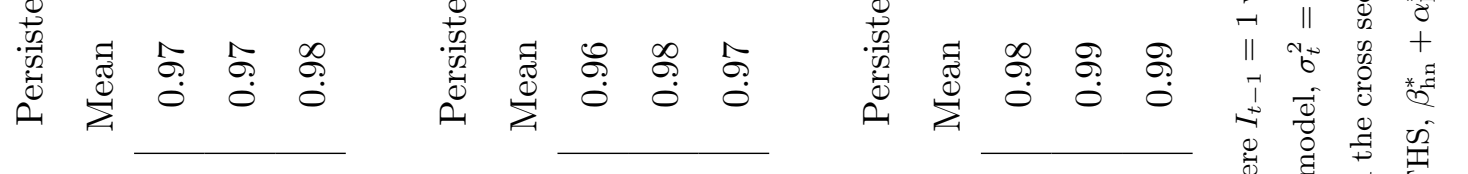

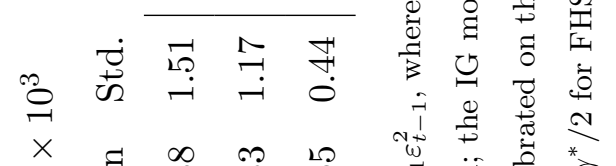

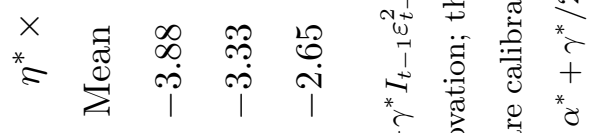

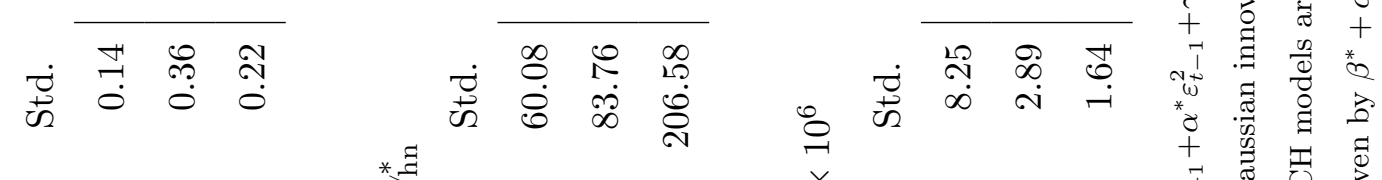
*

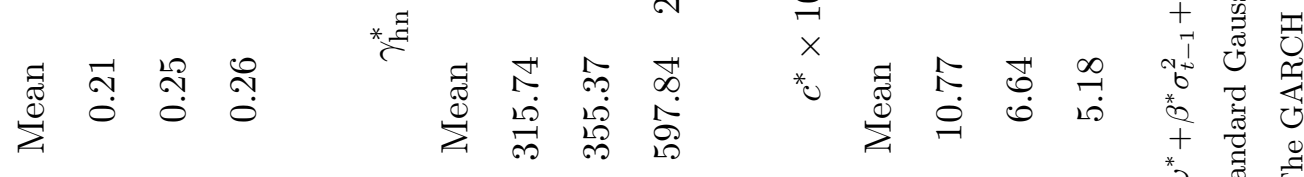

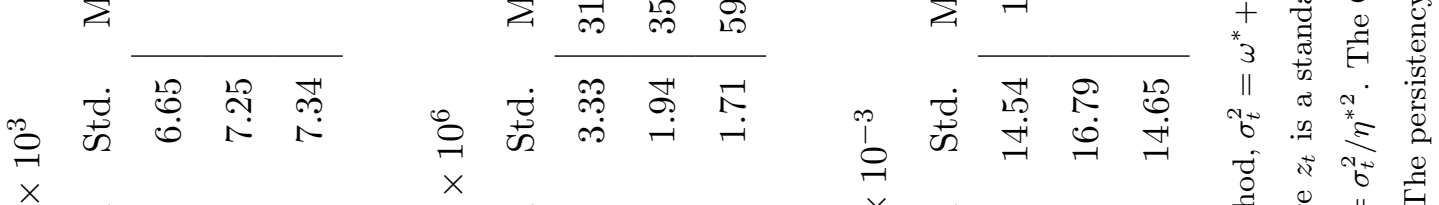

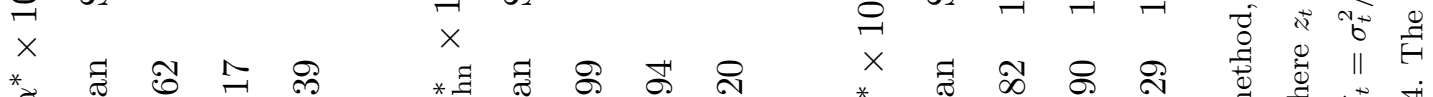

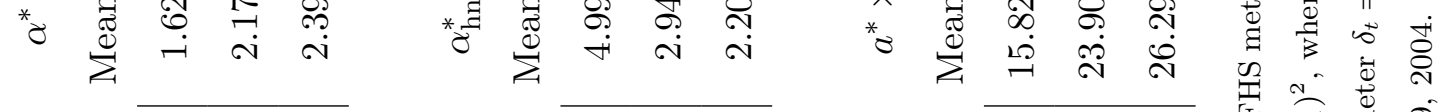

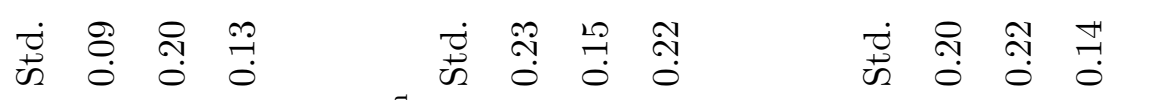

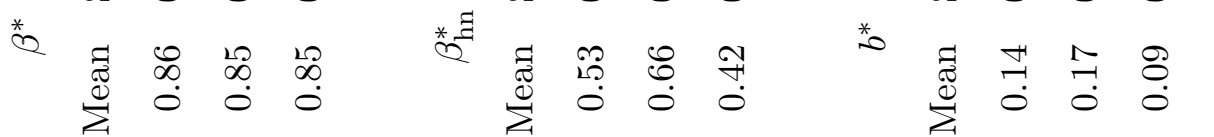

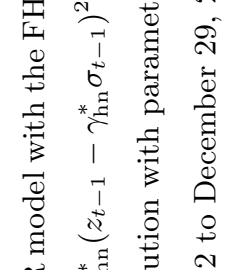

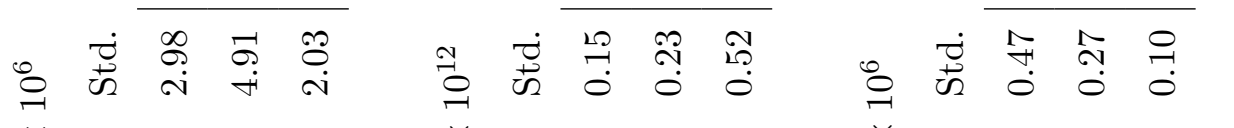

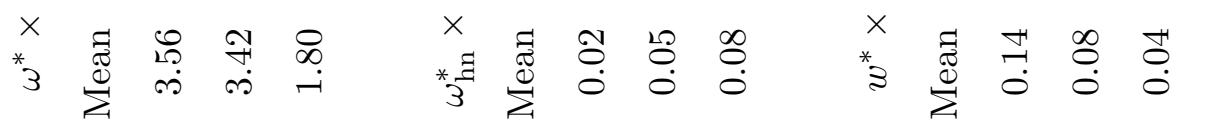

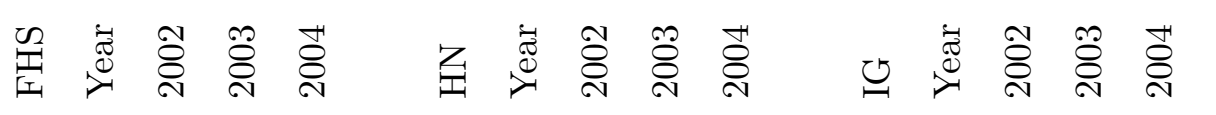

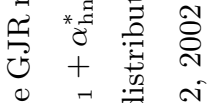

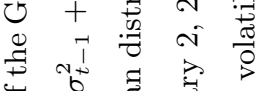

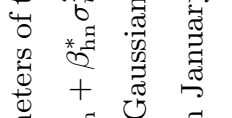

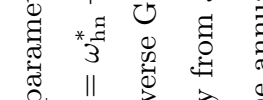

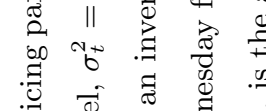

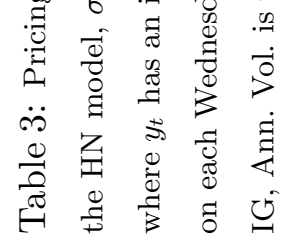




\begin{tabular}{lrrrrrr} 
& $a_{0}$ & $a_{1} \times 10^{3}$ & $a_{2} \times 10^{7}$ & $a_{3} \times 10$ & $a_{4} \times 10$ & $a_{5} \times 10^{4}$ \\
\hline 2002 & & & & & & \\
Mean & 1.11 & -1.41 & 6.23 & -1.42 & 2.57 & -2.02 \\
Std. & 0.12 & 0.22 & 1.09 & 1.37 & 1.41 & 1.83
\end{tabular}

2003

$\begin{array}{lrrrrrr}\text { Mean } & 1.07 & -1.45 & 6.48 & -1.88 & 1.64 & -0.57 \\ \text { Std. } & 0.21 & 0.31 & 1.32 & 2.52 & 1.19 & 3.12\end{array}$

2004

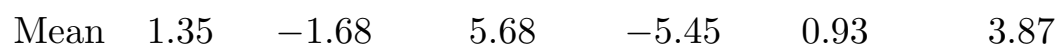

$\begin{array}{lllllll}\text { Std. } & 0.11 & 0.22 & 1.32 & 1.75 & 0.73 & 1.48\end{array}$

Table 4: Estimated coefficients of the ad hoc Black-Scholes model, $\sigma^{\text {bs }}=a_{0}+a_{1} K+a_{2} K^{2}+a_{3} \tau+a_{4} \tau^{2}+a_{5} K \tau$, where $\sigma^{\text {bs }}$ is the Black-Scholes implied volatility for an option with strike price $K$ and time to maturity $\tau$. The model is calibrated on the out-of-the-money SPX options on each Wednesday from January 2, 2002 to December 29, 2004. 
Panel A: Aggregate valuation errors across all years

\begin{tabular}{lrrrrrrrrr} 
& RMSE & MAE & MOE & Min & Max & Err $>0 \%$ & ErrBD\% & MAE\% & MOE\% \\
\hline FHS & 0.87 & 0.44 & 0.08 & -6.02 & 4.64 & 50.50 & 23.69 & 21.16 & 10.75 \\
HN & 1.19 & 0.74 & -0.20 & -5.66 & 6.47 & 30.65 & -44.20 & 23.93 & -25.74 \\
IG & 1.27 & 0.77 & -0.10 & -5.41 & 6.34 & 32.06 & -32.31 & 22.49 & -25.82 \\
BS & 3.39 & 2.15 & 0.06 & -15.88 & 28.66 & 50.82 & 69.95 & 57.91 & 39.46 \\
\hline
\end{tabular}

Panel B: Valuation errors by years

RMSE MAE MOE $\quad$ Min $\quad$ Max $\quad$ Err $>0 \% \quad$ ErrBD\% $\quad$ MAE\% $\quad$ MOE\%

\begin{tabular}{lrrrrrrrrr}
\hline 2002 & & & & & & & & & \\
FHS & 0.88 & 0.43 & 0.06 & -5.63 & 4.64 & 47.57 & 14.67 & 14.80 & 4.82 \\
HN & 1.16 & 0.72 & -0.21 & -4.64 & 5.55 & 27.59 & -48.00 & 19.55 & -33.06 \\
IG & 1.26 & 0.76 & -0.15 & -5.14 & 5.89 & 27.45 & -44.15 & 19.33 & -33.87 \\
BS & 3.69 & 2.36 & 0.03 & -15.25 & 28.66 & 48.38 & 51.20 & 58.95 & 39.79
\end{tabular}

2003

$\begin{array}{lrrrrrrrrr}\text { FHS } & 0.93 & 0.47 & -0.06 & -6.02 & 3.68 & 44.63 & 0.95 & 20.90 & 6.44 \\ \text { HN } & 0.92 & 0.57 & -0.28 & -3.82 & 4.92 & 25.73 & -64.94 & 22.10 & -32.13 \\ \text { IG } & 0.93 & 0.56 & -0.19 & -3.72 & 6.09 & 27.75 & -49.26 & 20.67 & -32.09 \\ \text { BS } & 3.32 & 2.12 & 0.03 & -15.88 & 15.35 & 49.86 & 68.37 & 58.59 & 38.69\end{array}$

2004

$\begin{array}{lrrrrrrrrr}\text { FHS } & 0.79 & 0.43 & 0.23 & -3.41 & 3.90 & 58.86 & 53.82 & 27.61 & 20.58 \\ \text { HN } & 1.41 & 0.91 & -0.12 & -5.66 & 6.47 & 38.24 & -21.06 & 29.92 & -12.60 \\ \text { IG } & 1.53 & 0.98 & 0.02 & -5.41 & 6.34 & 40.62 & -4.86 & 27.27 & -12.08 \\ \text { BS } & 3.15 & 1.98 & 0.12 & -13.98 & 15.18 & 54.11 & 89.74 & 56.26 & 39.85\end{array}$

Table 5: In-sample pricing errors of the different pricing models. RMSE is the root mean square error of the dollar pricing error (model price - market price); MAE is the dollar average absolute pricing error outside the bid-ask spread (MAE\% is in relative terms and in percentage); MOE is the dollar average pricing error outside the bid-ask spread (MOE\% is in relative terms and in percentage); Min (Max) is the minimum (maximum) pricing error; Err $>0 \%$ is the percentage of positive pricing errors; ErrBD\% is the average of $100 \times$ (model price - market price)/(bid-ask spread). 


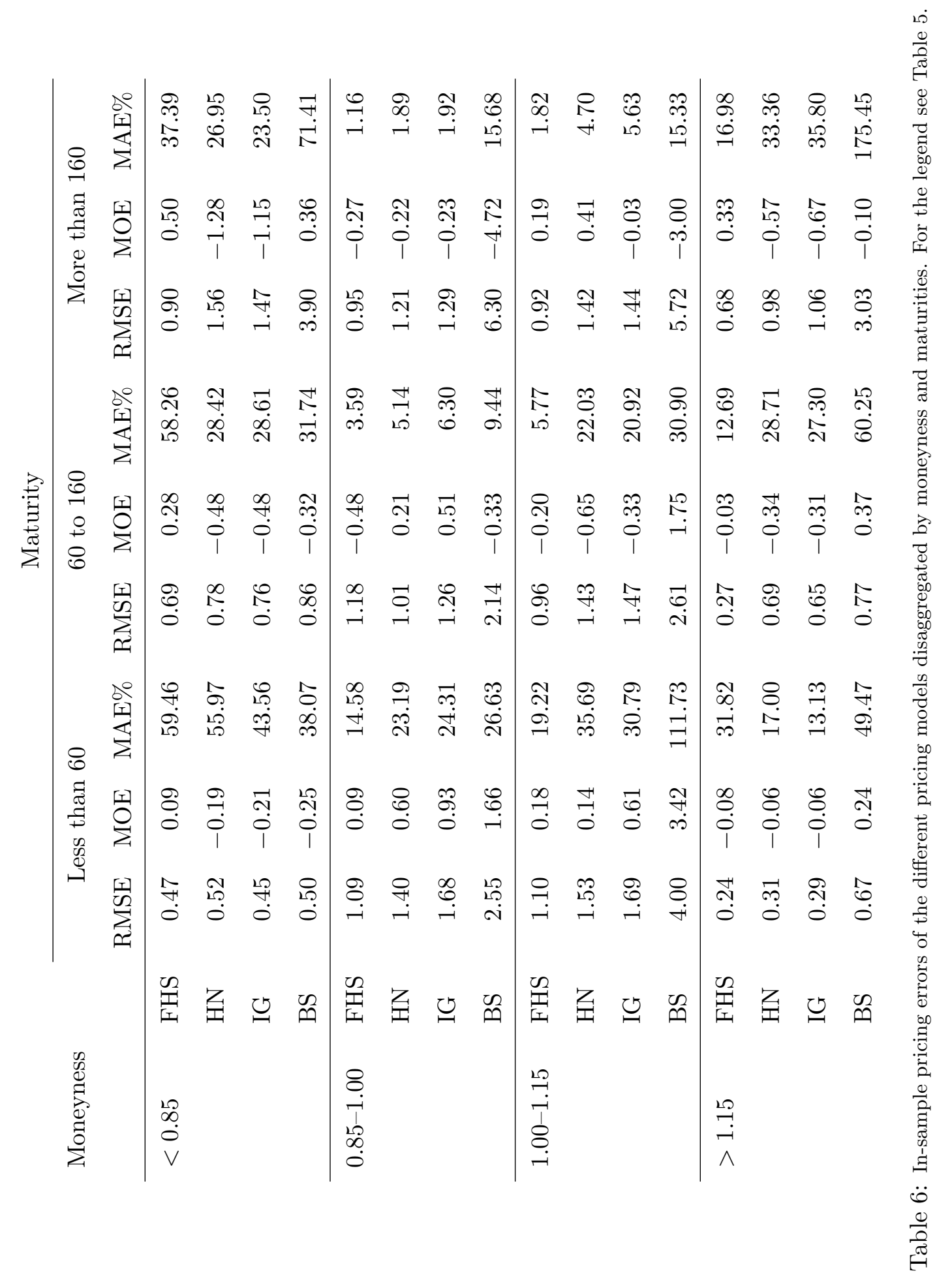


Panel A: Aggregate valuation errors across all years

\begin{tabular}{lrrrrrrrrr} 
& RMSE & MAE & MOE & Min & Max & Err $>0 \%$ & ErrBD\% & MAE\% & MOE\% \\
\hline FHS & 1.48 & 0.86 & 0.09 & -9.20 & 7.84 & 49.92 & 31.80 & 26.52 & 13.09 \\
HN & 1.55 & 0.98 & -0.20 & -7.69 & 9.52 & 30.27 & -44.90 & 25.36 & -25.38 \\
IG & 2.36 & 1.33 & 0.19 & -11.12 & 20.91 & 35.08 & 2.48 & 27.90 & -19.61 \\
BS & 3.82 & 2.32 & 0.18 & -18.19 & 63.31 & 50.97 & 88.89 & 66.38 & 47.03 \\
\hline
\end{tabular}

Panel B: Valuation errors by years

\begin{tabular}{lrrrrrrrrr} 
& RMSE & MAE & MOE & Min & Max & Err $>0 \%$ & ErrBD\% & MAE\% & MOE\% \\
\hline 2002 & & & & & & & & & \\
FHS & 1.72 & 1.00 & 0.03 & -9.20 & 7.10 & 43.61 & 18.01 & 19.64 & 5.57 \\
HN & 1.70 & 1.07 & -0.27 & -7.69 & 6.76 & 26.33 & -53.00 & 21.16 & -33.26 \\
IG & 2.22 & 1.36 & -0.09 & -11.12 & 12.65 & 29.11 & -35.65 & 22.68 & -31.56 \\
BS & 4.41 & 2.59 & 0.27 & -16.03 & 63.31 & 49.59 & 80.71 & 70.94 & 51.32 \\
& & & & & & & & & \\
2003 & & & & & & & & & \\
FHS & 1.36 & 0.78 & -0.14 & -8.16 & 6.06 & 41.12 & -10.62 & 23.28 & 4.81 \\
HN & 1.29 & 0.81 & -0.31 & -6.99 & 5.59 & 24.51 & -70.93 & 23.87 & -32.44 \\
IG & 1.58 & 0.96 & 0.01 & -6.52 & 9.50 & 30.02 & -31.94 & 22.81 & -29.44 \\
BS & 3.62 & 2.27 & 0.11 & -18.19 & 26.76 & 49.32 & 78.23 & 68.51 & 47.51 \\
& & & & & & & & & \\
2004 & & & & & & & & & \\
FHS & 1.35 & 0.81 & 0.37 & -7.12 & 7.84 & 63.86 & 83.99 & 35.76 & 27.62 \\
HN & 1.63 & 1.07 & -0.03 & -5.99 & 9.52 & 39.21 & -13.22 & 30.54 & -11.67 \\
IG & 3.00 & 1.65 & 0.63 & -7.08 & 20.91 & 45.19 & 69.07 & 37.38 & 0.37 \\
BS & 3.40 & 2.10 & 0.16 & -13.46 & 32.05 & 53.75 & 106.26 & 60.27 & 42.72 \\
\hline & & & & & & & & & \\
\hline
\end{tabular}

Table 7: Out-of-sample pricing errors of the different pricing models. For the legend see Table 5. 


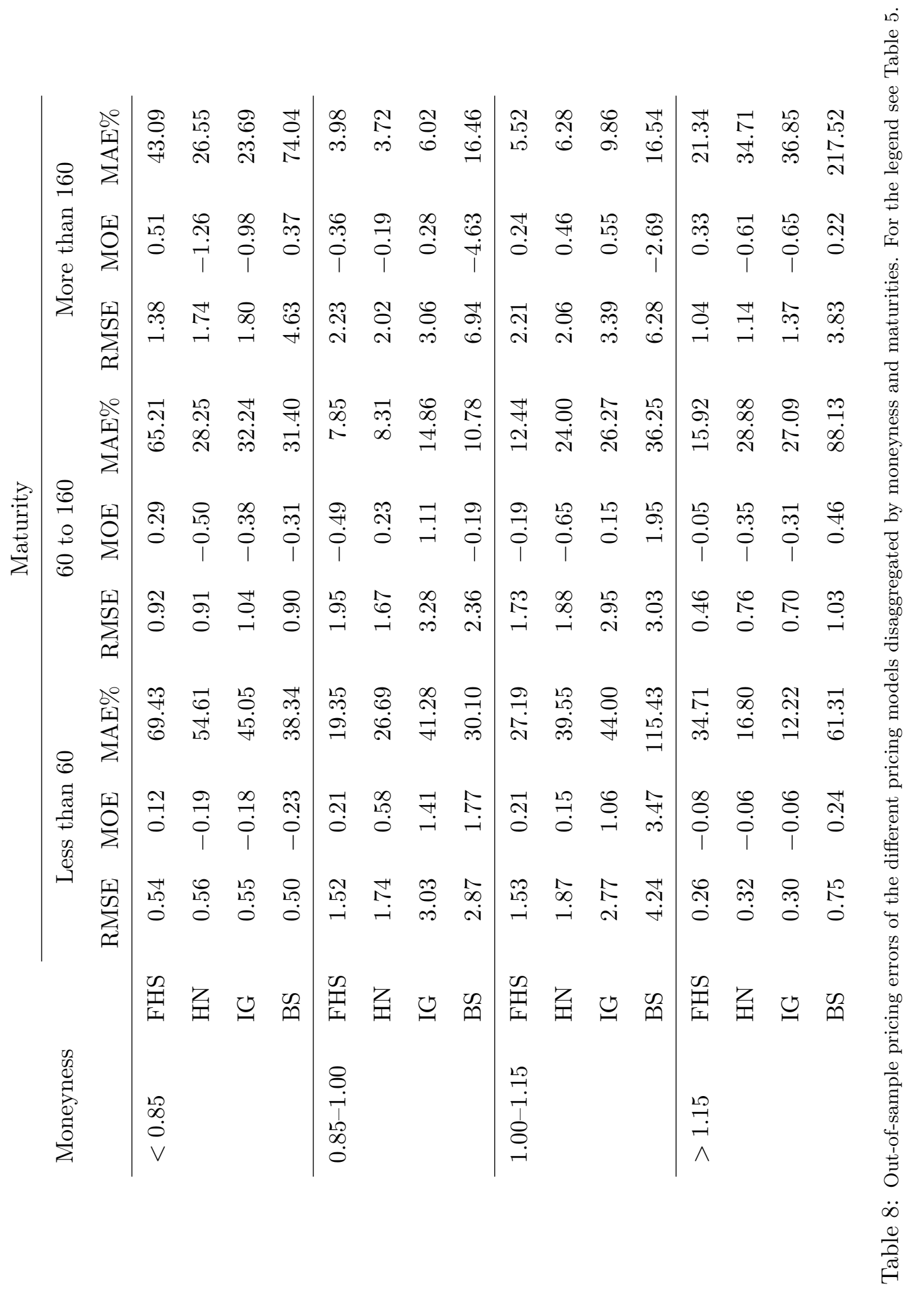


Panel A: In-sample aggregate valuation errors across all years

\begin{tabular}{lrrrrrrrrr} 
& RMSE & MAE & MOE & Min & Max & Err $>0 \%$ & ErrBD\% & MAE\% & MOE\% \\
\hline FHS & 0.87 & 0.44 & 0.08 & -6.02 & 4.64 & 50.50 & 23.69 & 21.16 & 10.75 \\
HIST & 3.32 & 1.88 & 0.22 & -19.64 & 15.29 & 48.57 & 35.82 & 31.38 & 8.46 \\
GAUSS & 0.99 & 0.53 & -0.23 & -6.44 & 4.72 & 32.38 & -18.85 & 16.72 & -2.10 \\
HN & 1.19 & 0.74 & -0.20 & -5.66 & 6.47 & 30.65 & -44.20 & 23.93 & -25.74 \\
\hline
\end{tabular}

Panel B: Out-of-sample aggregate valuation errors across all years

\begin{tabular}{lrrrrrrrrr} 
& RMSE & MAE & MOE & Min & Max & Err $>0 \%$ & ErrBD\% & MAE\% & MOE\% \\
\hline FHS & 1.48 & 0.86 & 0.09 & -9.20 & 7.84 & 49.92 & 31.80 & 26.52 & 13.09 \\
HIST & 3.33 & 1.87 & 0.18 & -18.55 & 16.06 & 48.56 & 29.66 & 31.18 & 0.31 \\
GAUSS & 1.55 & 0.90 & -0.22 & -10.13 & 9.03 & 37.35 & -9.43 & 22.13 & -8.26 \\
HN & 1.55 & 0.98 & -0.20 & -7.69 & 9.52 & 30.27 & -44.90 & 25.36 & -25.38 \\
\hline
\end{tabular}

Table 9: In- and out-of-sample pricing errors of the different pricing models. FHS is the GJR GARCH model with FHS innovations calibrated on the cross-section of out-of-the-money SPX option prices; HIST is the GJR GARCH model with FHS innovations estimated on historical returns; GAUSS is the GJR GARCH model with Gaussian innovations calibrated on the cross-section of out-of-the-money SPX option prices; HN is the Heston-Nandi GARCH model with Gaussian innovations calibrated on the cross-section of out-of-the-money SPX option prices. For the legend see Table 5.

\begin{tabular}{lccccc}
$\mathrm{S} \& \mathrm{P} 500$ & 800 & 900 & 1,000 & 1,100 & 1,200 \\
\hline$M_{t, t+38}$ & 1.72 & 1.26 & 1.08 & 0.64 & 0.20 \\
$M_{t, t+73}$ & 1.37 & 1.27 & 1.15 & 0.71 & 0.36 \\
$M_{t, t+164}$ & 1.56 & 1.48 & 1.22 & 0.84 & 0.48 \\
$M_{t, t+255}$ & 1.69 & 1.64 & 1.26 & 0.90 & 0.59
\end{tabular}

Table 10: State price densities per unit probability, $M_{t, t+\tau}$, for different time to maturities $\tau=38,73,164,255$ days, estimated on July 9, 2003; see also Figure 5. $M_{t, t+\tau}$ is the discounted ratio of the pricing over the historical densities of the GJR GARCH model with FHS innovations. 


\begin{tabular}{rrr|rr} 
& \multicolumn{2}{c|}{ FHS } & \multicolumn{2}{c}{ GAUSS } \\
$k / S_{t}$ & $M_{t, T}$ & $R_{t, T} \%$ & $M_{t, T}$ & $R_{t, T} \%$ \\
\cline { 2 - 5 } 0.40 & 1.50 & -33.3 & 4.20 & -76.2 \\
0.60 & 1.45 & -31.0 & 2.80 & -64.3 \\
0.80 & 1.40 & -28.6 & 1.80 & -44.4 \\
1.00 & 1.20 & -16.7 & 1.20 & -16.7 \\
1.20 & 0.90 & 11.1 & 0.90 & 11.1
\end{tabular}

Table 11: Average state price densities per unit probability, $M_{t, T}$, for long maturities in 2002, in our database; see also Figure 6. $M_{t, T}$ is the discounted ratio of the pricing over the historical densities of the GJR GARCH model with FHS and Gaussian innovations, respectively. $R_{t, T}$ is the expected rate of return at time $t$ of an Arrow-Debreu security paying one dollar at time $T$ if $S_{T} \in(k, k+d k)$ and zero otherwise. 

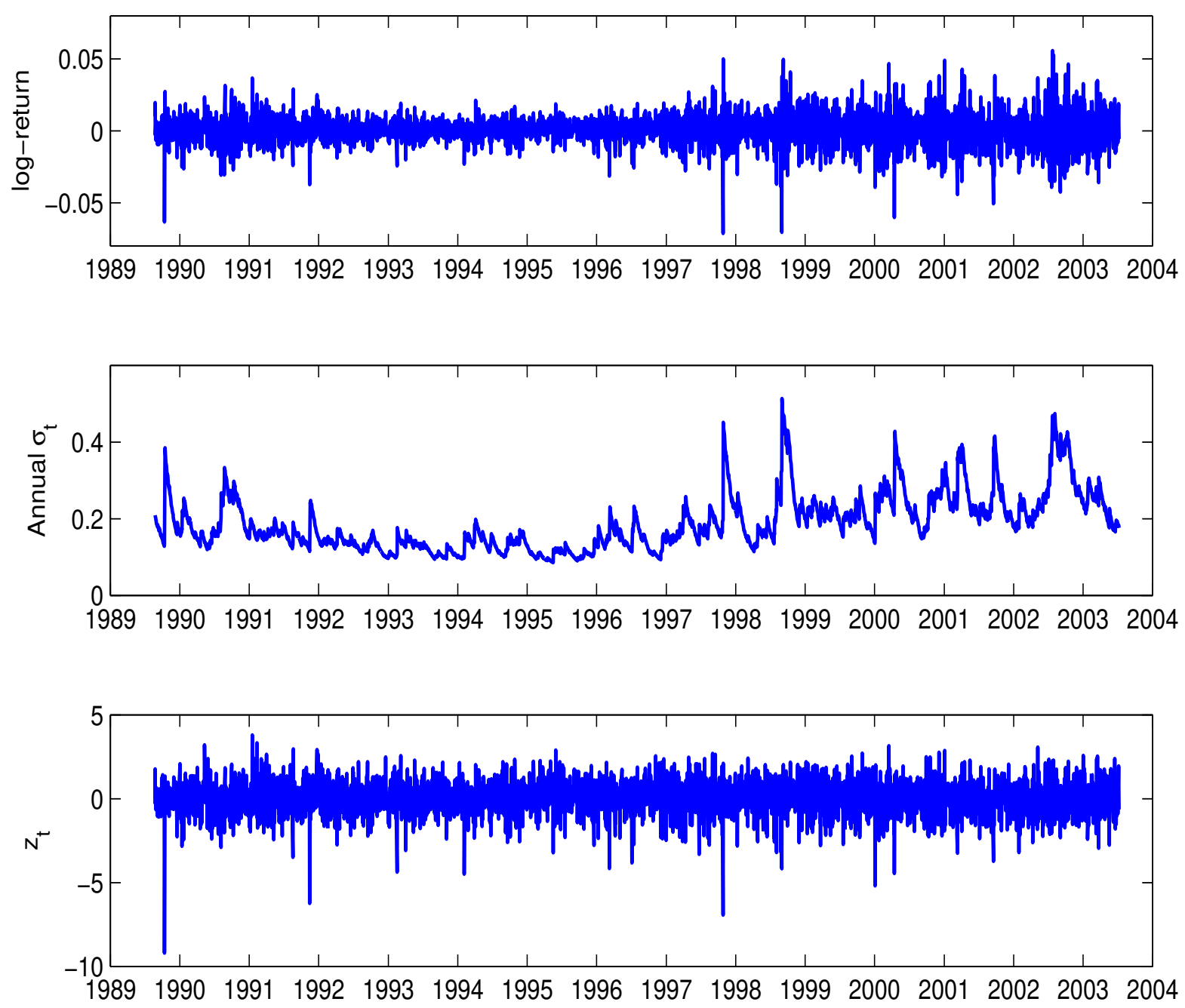

Figure 1: Daily log-returns of the S\&P 500 index from August 23, 1989 to July 9, 2003, (i.e. 3,500 log-returns), conditional GARCH volatility $\sigma_{t}$ (on an annual base) and FHS innovations $z_{t}$. The GARCH model is estimated using the PML method with the nominal assumption of Gaussian innovations 


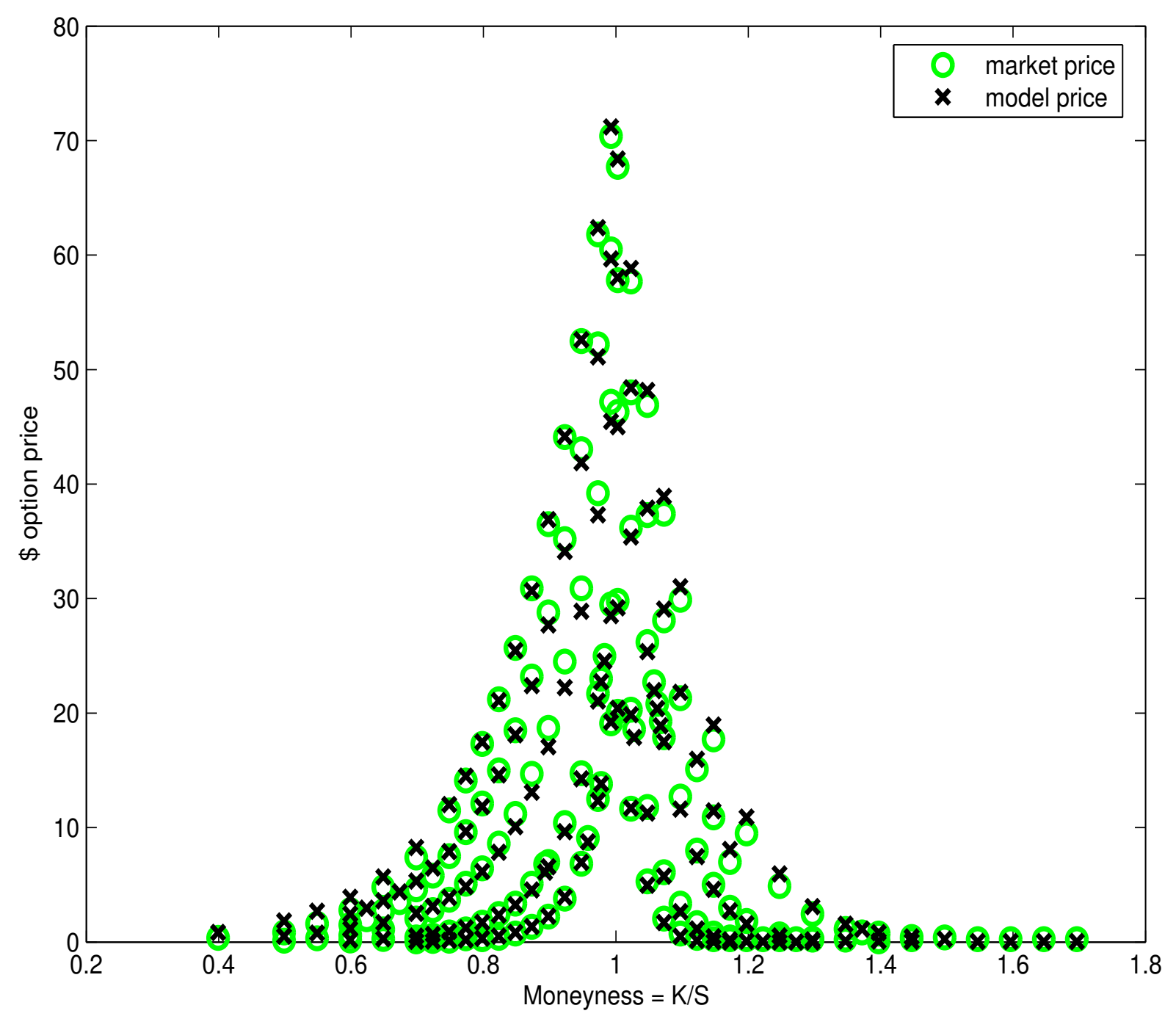

Figure 2: Calibration of the GJR GARCH model with the FHS method on the cross section of out-of-the-money SPX options on July 9, 2003. Moneyness is the strike price $K$ divided by the spot asset price $S$. 

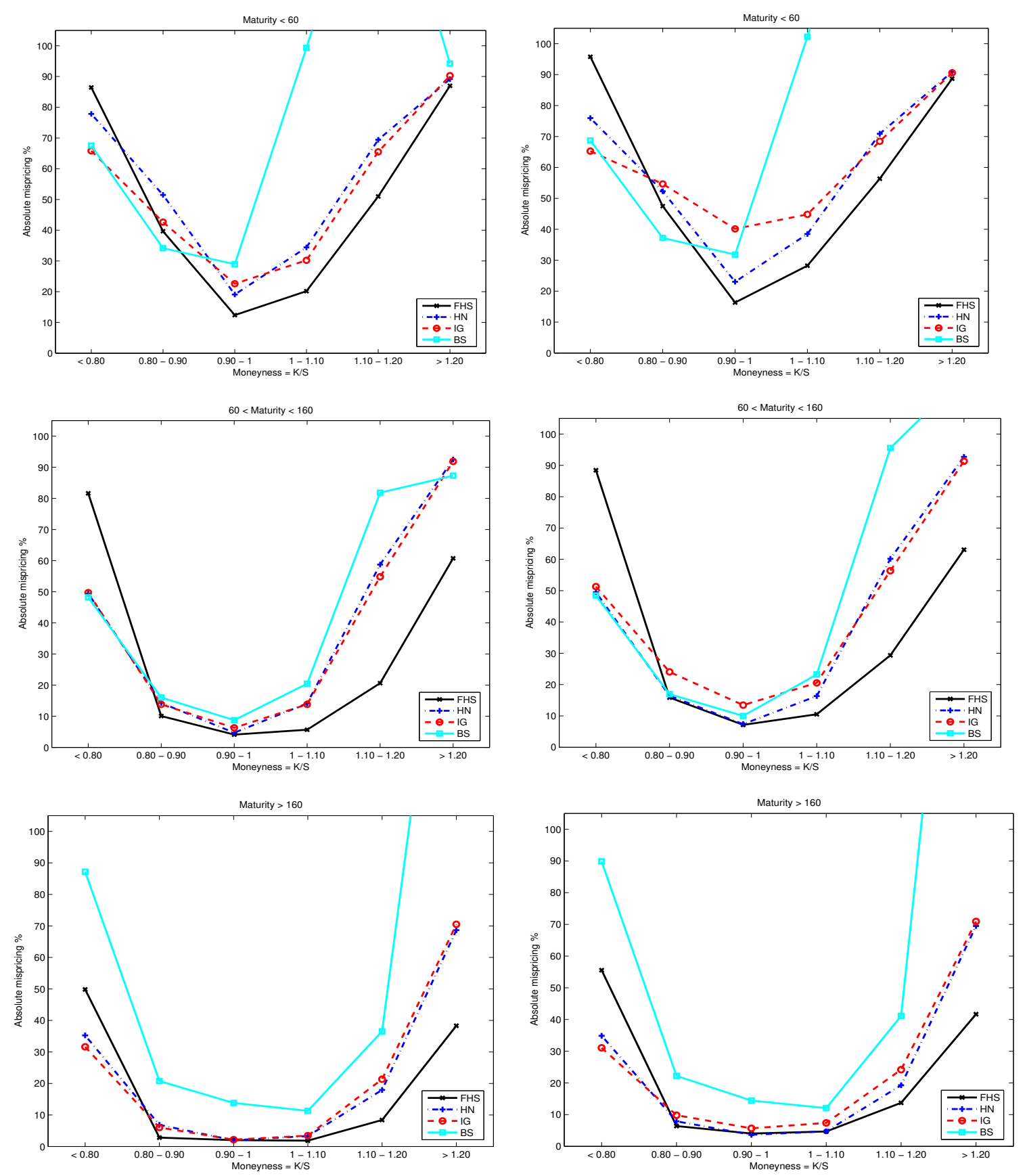

Figure 3: Absolute mispricing in percentage, i.e. $100 \times \mid$ model price - market price $\mid /$ market price, of the different pricing models, averaged across Wednesdays from January 2, 2002 to December 29, 2004 for out-of-the-money SPX options. Left graphs are in-sample comparisons and right graphs are out-of-sample comparisons. Moneyness is the strike price $K$ divided by the spot asset price $S$. 

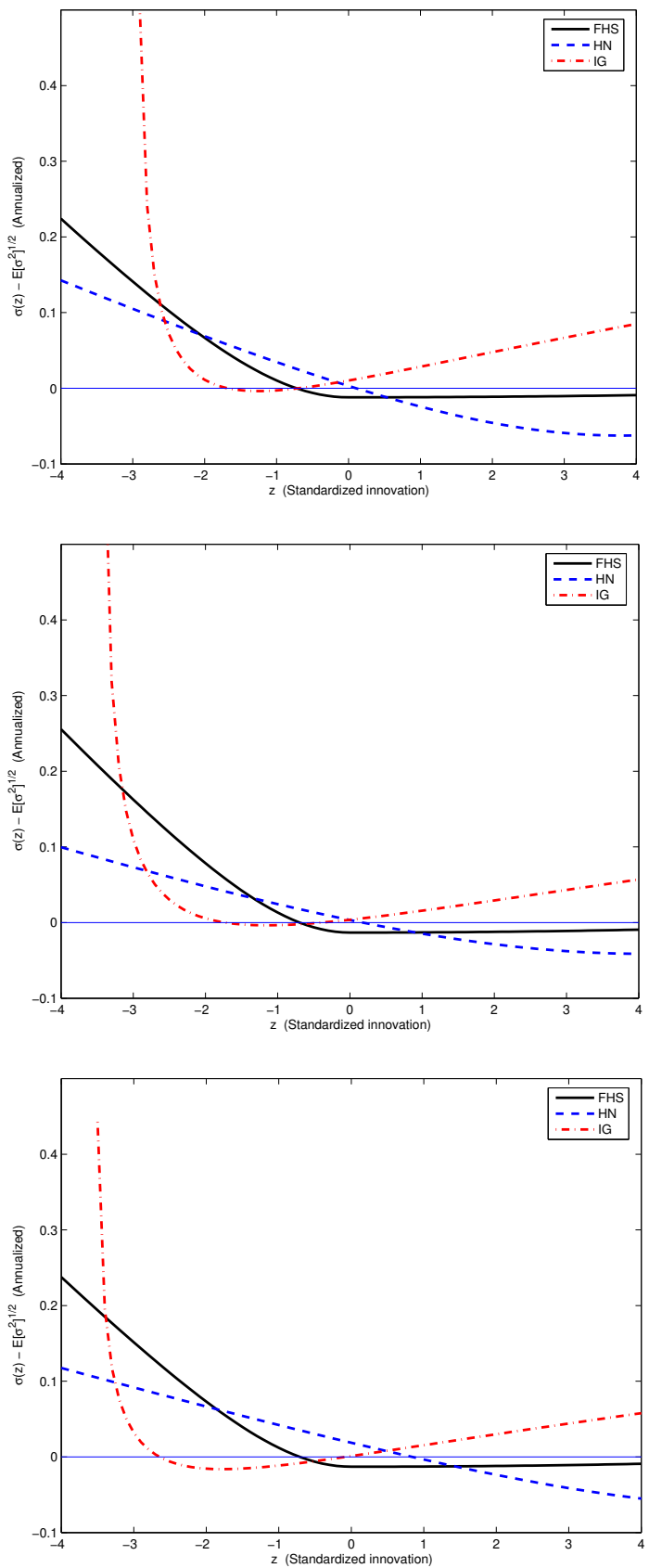

Figure 4: News impact curves of the FHS, HN and IG GARCH models based on the average pricing parameters reported in Table 3 for 2002, 2003 and 2004 from top to bottom. The horizontal axis is the standardized GARCH innovations $z$. The vertical axis is the change in volatility from the long-run level, $\sigma(z)-\sqrt{E_{\mathbb{Q}}\left[\sigma^{2}\right]}$ on an annual base as a function of the standardized innovation $z$. For GJR model $\sigma^{2}(z)=\omega^{*}+\beta^{*} E_{\mathbb{Q}}\left[\sigma^{2}\right]+E_{\mathbb{Q}}\left[\sigma^{2}\right]\left(\alpha^{*}+\gamma^{*} I_{\{z<0\}}\right) z^{2}$; for HN model $\sigma^{2}(z)=\omega_{\mathrm{hn}}^{*}+\beta_{\mathrm{hn}}^{*} E_{\mathbb{Q}}\left[\sigma^{2}\right]+\alpha_{\mathrm{hn}}^{*}\left(z-\gamma_{\mathrm{hn}}^{*} \sqrt{E_{\mathbb{Q}}\left[\sigma^{2}\right]}\right)^{2}$; for IG model $\sigma^{2}(z)=w^{*}+b^{*} E_{\mathbb{Q}}\left[\sigma^{2}\right]+c^{*} \tilde{y}(z)+a^{*} E_{\mathbb{Q}}\left[\sigma^{2}\right]^{2} / \tilde{y}(z)$, where $\tilde{y}(z)=\left(z+\sqrt{\delta^{*}}\right) \sqrt{\delta^{*}}$, and $\delta^{*}=E_{\mathbb{Q}}\left[\sigma^{2}\right] / \eta^{* 2}$. Notice that $\tilde{y}(z)$ is the positive unscaled inverse Gaussian innovation. 

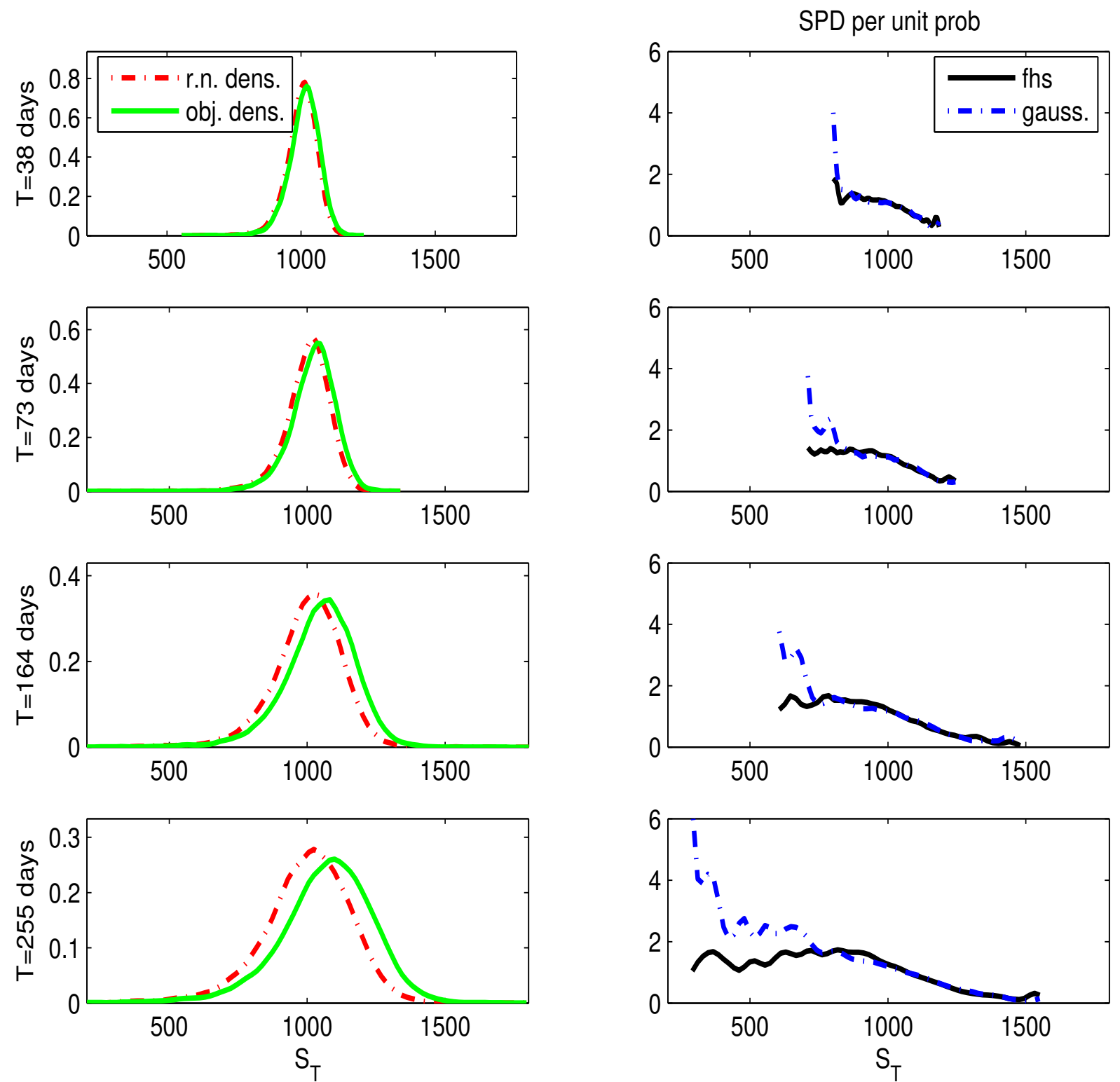

Figure 5: Left graphs: pricing and historical density estimates for different time horizons on July 9, 2003. The pricing density is obtained by calibrating the GJR model with the FHS method on the cross-section of out-of-the-money SPX options. The historical density is obtained by fitting the GJR GARCH model to 3,500 historical S\&P 500 log-returns and using the PML method. Right graphs: SPD per unit probability given by the discounted ratio of the pricing over the historical densities. The Gaussian SPD (gauss.) are based on the GJR GARCH model with Gaussian innovations calibrated on the cross-section of out-of-the-money SPX options. 

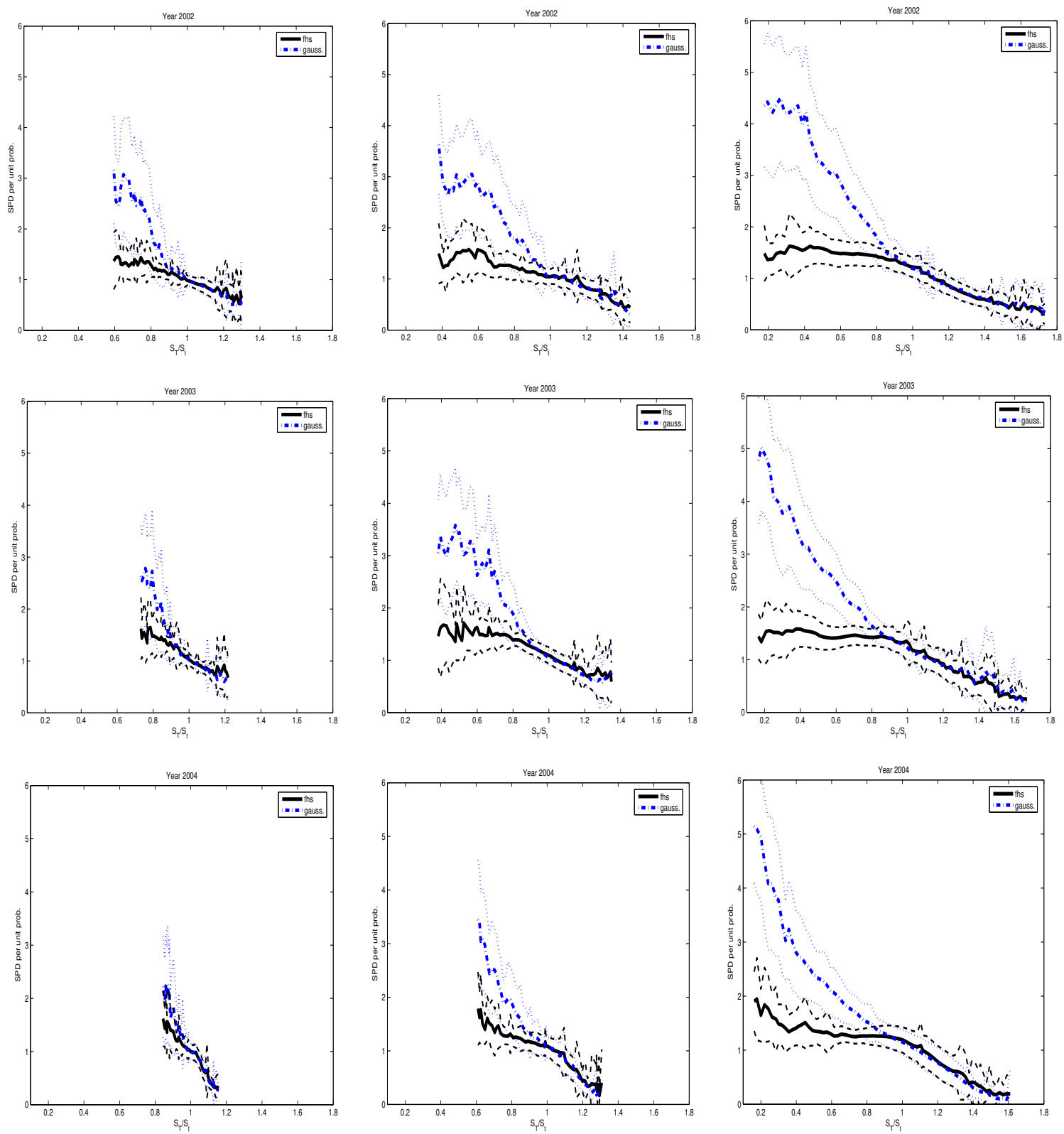

Figure 6: Average SPD per unit probability for 2002, 2003 and 2004 (from top to bottom), and short, medium and long maturities (from left to right). For each Wednesday $t$ from January 2, 2002 to December 29, 2004 and for each time to maturity $\tau$ between 10 and 360 days, the SPD per unit probability, $M_{t, t+\tau}$, are estimated by the discounted ratio of the pricing over the historical densities. For each Wednesday $t$, the pricing GJR model is calibrated on the cross-section of out-of-the-money SPX options, and the historical GJR model is estimated using 3,500 historical S\&P 500 log-returns. Two estimates of $M_{t, t+\tau}$ are provided using ( $i$ ) the FHS method (fhs) and ( $i i$ ) the GAUSS method (gauss.). In case ( $i$ ) historical and pricing GJR models are simulated using FHS innovations, and in case (ii) using Gaussian innovations. For each year and each maturity category, the graphs show the average $M_{t, T}$ computed for a given set of gross returns $S_{T} / S_{t}$, and the average $M_{t, T} \pm 0.5$ times the empirical standard deviation of $M_{t, T}$. 

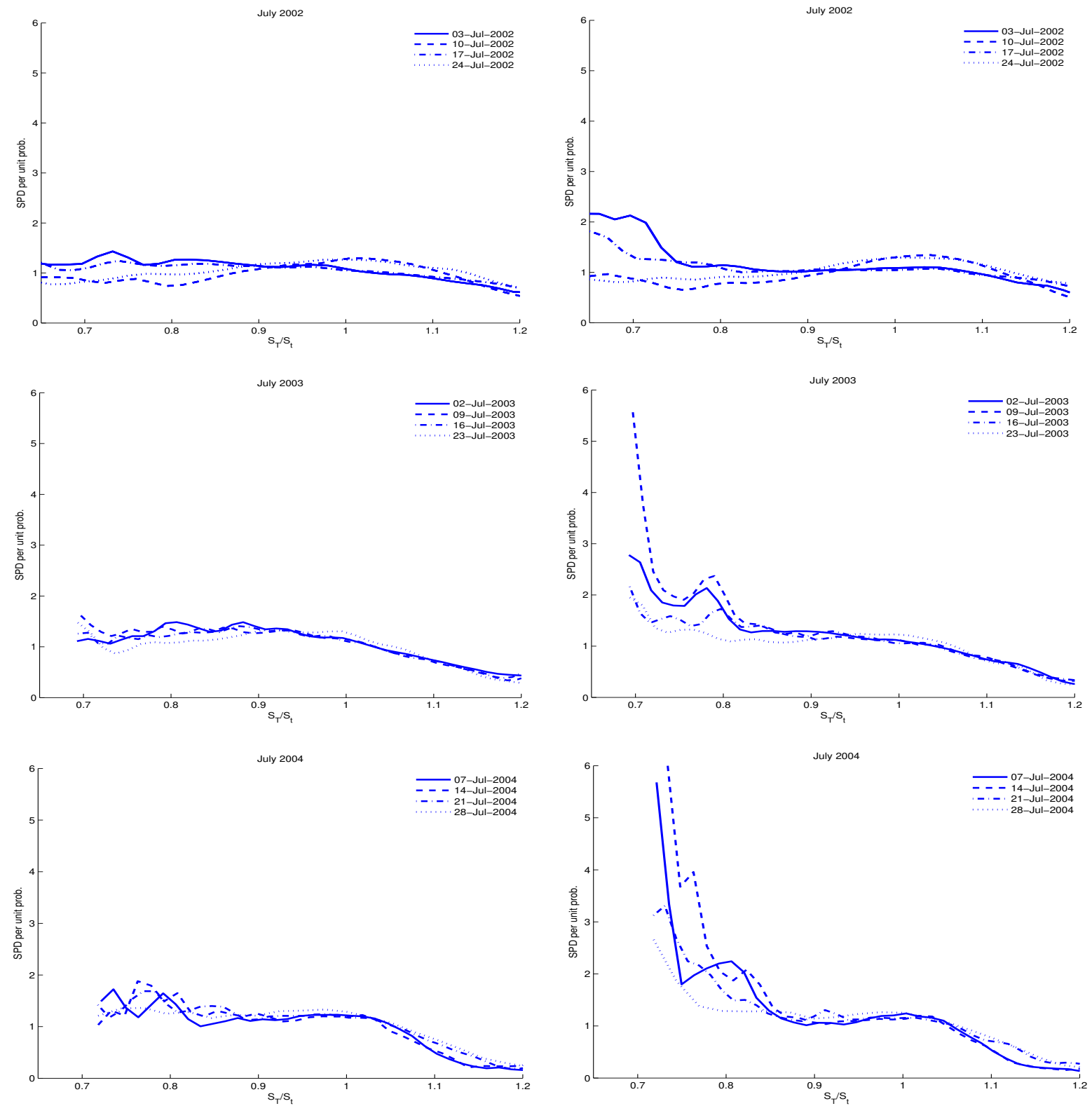

Figure 7: SPD per unit probability on four consecutive Wednesdays in July 2002, July 2003 and July 2004 and for time to maturity $\tau$ closest to 100 days. SPD per unit probability, $M_{t, t+\tau}$, are estimated by the discounted ratio of the pricing over the historical densities. For each Wednesday $t$, the pricing GJR model is calibrated on the cross-section of out-of-the-money SPX options, and the historical GJR model is estimated using 3,500 historical S\&P 500 log-returns. Two estimates of $M_{t, t+\tau}$ are provided using $(i)$ the FHS method (left graphs) and (ii) the GAUSS method (right graphs). In case $(i)$ historical and pricing GJR models are simulated using FHS innovations, and in case ( $i i)$ using Gaussian innovations. 


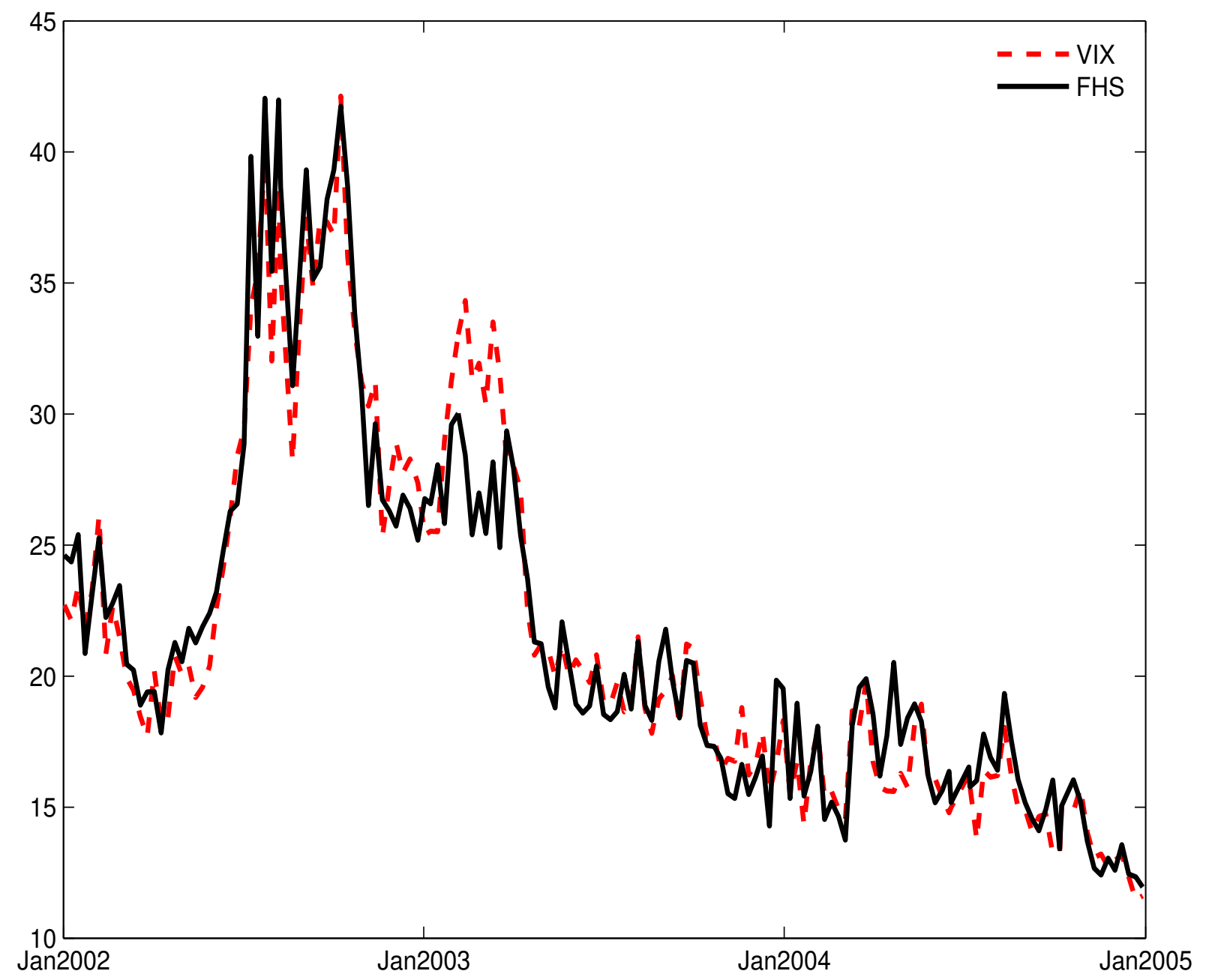

Figure 8: For each Wednesday $t$ from January 2, 2002 to December 29, 2004, the graph shows VIX 30-day volatility index at time $t$ and conditional forecast of the risk neutral integrated volatility, $100 \times \sqrt{(365 / 30) \sum_{i=1}^{30} E_{\mathbb{Q}}\left[\sigma_{t+i}^{2} \mid \mathcal{F}_{t}\right]}$, given by the GJR model with the FHS method. 